\title{
Deficient nitric oxide signalling impairs skeletal muscle growth and performance: involvement of mitochondrial dysregulation
}

Clara De Palma ${ }^{1}$, Federica Morisi ${ }^{1}$, Sarah Pambianco ${ }^{1}$, Emma Assi ${ }^{2}$, Thierry Touvier $^{1}$, Stefania Russo ${ }^{2}$, Cristiana Perrotta ${ }^{1}$, Vanina Romanello ${ }^{3}$, Silvia Carnio ${ }^{3}$, Valentina Cappello ${ }^{4,5}$, Paolo Pellegrino ${ }^{1}$, Claudia Moscheni ${ }^{6}$, Maria Teresa Bassi ${ }^{2}$, Marco Sandri ${ }^{3,7}$, Davide Cervia ${ }^{1,8^{*}}$ and Emilio Clementi ${ }^{1,2^{*}}$

\begin{abstract}
Background: Nitric oxide (NO), generated in skeletal muscle mostly by the neuronal NO synthases (nNOS $\mu$ ), has profound effects on both mitochondrial bioenergetics and muscle development and function. The importance of NO for muscle repair emerges from the observation that nNOS signalling is defective in many genetically diverse skeletal muscle diseases in which muscle repair is dysregulated. How the effects of NO/nNOS $\mu$ on mitochondria impact on muscle function, however, has not been investigated yet.
\end{abstract}

Methods: In this study we have examined the relationship between the NO system, mitochondrial structure/activity and skeletal muscle phenotype/growth/functions using a mouse model in which nNOS $\mu$ is absent. Also, NO-induced effects and the NO pathway were dissected in myogenic precursor cells.

Results: We show that nNOS $\mu$ deficiency in mouse skeletal muscle leads to altered mitochondrial bioenergetics and network remodelling, and increased mitochondrial unfolded protein response (UPR ${ }^{\mathrm{mt}}$ ) and autophagy. The absence of nNOS $\mu$ is also accompanied by an altered mitochondrial homeostasis in myogenic precursor cells with a decrease in the number of myonuclei per fibre and impaired muscle development at early stages of perinatal growth. No alterations were observed, however, in the overall resting muscle structure, apart from a reduced specific muscle mass and cross sectional areas of the myofibres. Investigating the molecular mechanisms we found that nNOS $\mu$ deficiency was associated with an inhibition of the Akt-mammalian target of rapamycin pathway. Concomitantly, the Akt-FoxO3mitochondrial E3 ubiquitin protein ligase 1 (Mul-1) axis was also dysregulated. In particular, inhibition of nNOS/NO/cyclic guanosine monophosphate (cGMP)/cGMP-dependent-protein kinases induced the transcriptional activity of FoxO3 and increased Mul-1 expression. nNOS $\mu$ deficiency was also accompanied by functional changes in muscle with reduced muscle force, decreased resistance to fatigue and increased degeneration/damage post-exercise.

Conclusions: Our results indicate that nNOS $\mu / N O$ is required to regulate key homeostatic mechanisms in skeletal muscle, namely mitochondrial bioenergetics and network remodelling, UPR ${ }^{\mathrm{mt}}$ and autophagy. These events are likely associated with nNOS $\mu$-dependent impairments of muscle fibre growth resulting in a deficit of muscle performance.

Keywords: Nitric oxide synthase and signalling, Mitochondrial bioenergetics, Mitochondrial network, Unfolded protein response, Autophagy, Akt-mTOR pathway, Akt-FoxO3-Mul-1 axis, Fibre growth, Muscle structure, Muscle exercise

\footnotetext{
*Correspondence: d.cervia@unitus.it; emilio.clementi@unimi.it

'Unit of Clinical Pharmacology, National Research Council-Institute of Neuroscience, Department of Biomedical and Clinical Sciences "Luigi Sacco", University Hospital "Luigi Sacco", Università di Milano, Milano, Italy

${ }^{2}$ Scientific Institute IRCCS Eugenio Medea, Bosisio Parini, Italy

Full list of author information is available at the end of the article
} 


\section{Background}

Nitric oxide (NO) is a gas and a messenger with pleiotropic functions in most tissues and organs, synthesized by a family of NO synthases. NO is also generated in skeletal muscle, in particular by the muscle-specific neuronal NO synthases (nNOS or NOS1) [1,2]. nNOS $\mu$ is the predominant nNOS isoform in muscle and is anchored to the sarcolemma as a component of the dystrophin glycoprotein complex [3]. This enzyme produces $\mathrm{NO}$ at low, physiological levels (in the pico to nanomolar range) in a way controlled by second messengers $[1,2]$; its expression is increased by crush injury, muscle activity and ageing $[4,5]$. NO has an important role in regulating skeletal muscle physiological activity, including excitation-contraction coupling, muscle force generation, auto-regulation of blood flow, calcium homeostasis, metabolism and bioenergetics [2,6,7]. In addition, it is a key determinant in myogenesis that it regulates at several key steps, especially when the process is stimulated to repair muscle damage after injury $[5,8,9]$.

The importance of $\mathrm{NO}$ in muscle repair also emerges from the observation that nNOS signalling is defective in many genetically diverse skeletal muscle diseases in which muscle repair is dysregulated, including Duchenne muscular dystrophy, Becker muscular dystrophy, limb-girdle muscular dystrophies 2C, 2D and 2E, Ullrich congenital muscular dystrophy and inflammatory myositis [3,10-13]. Based on this evidence and on the fact that the restoration of NO signalling by nNOS overexpression ameliorates muscle function $[14,15]$, genetic and pharmacologic strategies to boost nNOS/NO signalling in dystrophic muscle are being tested with encouraging results: in particular, the combination of NO donation with non steroidal antiinflammatory activity limits muscle damage and favours muscle healing in vivo [16-18] such that it is currently being tested as a therapeutic for Duchenne muscular dystrophy in humans $[19,20]$.

The observation that nNOS is localised in close proximity to mitochondria suggests a tight coupling between $\mathrm{NO}$ generation and regulation of mitochondrial respiration and metabolism. The role of NO in regulating oxidative phosphorylation and mitochondrial biogenesis in skeletal muscle physiology has been established [21-24]. Likewise NO-dependent inhibition of mitochondrial fission occurs during myogenic differentiation [25].

How the effects of NO on mitochondria impact on muscle function, however, has not been investigated yet. Elucidation of this aspect is relevant in view of the role that mitochondria play in muscle pathophysiology and may shed light on the muscular disorders in which $\mathrm{NO}$ signalling is impaired [26]. In particular, increases in mitochondria number and oxidative phosphorylation activity is relevant during differentiation [27] and the balance of fission and fusion is necessary to preserve excitation contraction coupling and prevent atrophy $[28,29]$. In addition, mitochondria are involved in regulating autophagy [30], whose derangement plays a role in a number of inherited muscle diseases [31-33]. Mitochondrial protein homeostasis is maintained through proper folding and assembly of polypeptides. This involves the mitochondrial unfolded protein response $\left(\mathrm{UPR}^{\mathrm{mt}}\right)$, a stress response that activates transcription of nuclear-encoded mitochondrial chaperone genes to maintain proteins in a folding or assembly-competent state, preventing deleterious protein aggregation [34-36].

In this study we have examined the relationship between the NO system, mitochondrial structure/activity and skeletal muscle phenotype/growth/functions using a mouse model in which nNOS $\mu$ is absent (NOS1-/-). Also, NOinduced effects and the NO pathway were dissected in myogenic precursor cells. Our results indicate that the deficit in NO signalling leads in skeletal muscle to alterations in mitochondrial morphology, bioenergetics and network remodelling, accompanied by defective autophagy and the induction of a UPR ${ }^{\mathrm{mt}}$ response. These events, while not severely altering the overall resting skeletal muscle structure, are associated with modifications in the Akt-mammalian target of rapamycin (mTOR) pathway and Akt-FoxO3-mitochondrial E3 ubiquitin protein ligase 1 (Mul-1) axis and are sufficient to dysregulate skeletal muscle growth and exercise performance.

\section{Methods}

\section{Animals}

NOS1-/- animals are mice homozygous for targeted disruption of the nNOS gene (strain name B6129S4$\left.\mathrm{NOS}^{\mathrm{tm} 1 \mathrm{Plh}} / \mathrm{J}\right)$ that were purchased from Jackson Laboratories (Bar Harbor, Maine, USA) (stock no. 002633). In this mouse line, targeted deletion of exon 2 specifically eliminates expression of $n N O S \mu$ [37]. NOS1-/- mice were crossed with the wild-type B6129 to maintain the original background and to obtain a colony of NOS1-/- mice and wild-type littermate controls, with genotyping performed from tail clippings. Experiments were performed on male mice at postnatal day 10 (P10) and P120. C57BL/6 wildtype mice (strain name C57Bl10SnJ) were purchased from Charles River (Calco, Italy). Animals were housed in a regulated environment $\left(23 \pm 1^{\circ} \mathrm{C}, 50 \pm 5 \%\right.$ humidity $)$ with a 12-hour light/dark cycle (lights on at 08.00 a.m.), and provided with food and water ad libitum. For specific experiments, mice were killed by cervical dislocation. All studies were conducted in accordance with the Italian law on animal care $N^{\circ} 116 / 1992$ and the European Communities Council Directive EEC/609/86. The experimental protocols were approved by the Ethics Committee of the University of Milano. All efforts were made to reduce both animal suffering and the number of animals used. 


\section{Mitochondrial membrane potential}

Mitochondrial membrane potential in isolated transfected fibres from flexor digitorum brevis muscles was measured by epifluorescence microscopy based on the accumulation of tetramethylrhodamine methyl ester (TMRM) fluorescence $[25,29,38]$. Briefly, flexor digitorum brevis myofibres were placed in $1 \mathrm{ml}$ Tyrode's buffer and loaded with $5 \mathrm{nM}$ TMRM supplemented with $1 \mu \mathrm{M}$ cyclosporine $\mathrm{H}$ for 30 minutes at $37^{\circ} \mathrm{C}$. Myofibres were then observed with an Olympus IX81 inverted microscope equipped with a CellR imaging system (Olympus, Tokio, Japan). Sequential images of TMRM fluorescence were acquired every 60 seconds with $\mathrm{a} \times 200.5$, UPLANSL $\mathrm{N}$ A objective (Olympus). When indicated, oligomycin $(5 \mu \mathrm{M})$ or the protonophore carbonylcyanide-p-trifluoromethoxyphenyl hydrazone (FCCP, $4 \mu \mathrm{M}$ ) was added [39]. Images were acquired and stored, and analysis of TMRM fluorescence over mitochondrial regions of interest was performed using ImageJ software (http://rsbweb.nih.gov/ij/).

\section{Primary myogenic cell cultures}

Using published protocols [25], myogenic precursor cells (satellite cells) were freshly isolated from the muscles of newborn C57BL/6 mice. When indicated, cells were obtained from NOS1-/- mice and wild-type littermate controls. Briefly, hind limb muscles were digested with $2 \%$ collagenase-II and dispase for 10 minutes at $37^{\circ} \mathrm{C}$ with gentle agitation. Contamination by non-myogenic cells was reduced by pre-plating the collected cells onto plastic dishes where fibroblasts tend to adhere more rapidly. Dispersed cells were then resuspended in Iscove's modified Dulbecco's medium supplemented with $20 \%$ foetal bovine serum, $3 \%$ chick embryo extract (custom made), $10 \mathrm{ng} / \mathrm{ml}$ fibroblast growth factor, $100 \mathrm{U} / \mathrm{ml}$ penicillin, $100 \mu \mathrm{g} / \mathrm{ml}$ streptomycin and $50 \mu \mathrm{g} / \mathrm{ml}$ gentamycin, and plated onto matrigel-coated dishes. Differentiation was induced by changing the medium to Iscove's modified Dulbecco's medium supplemented with $2 \%$ horse serum and the antibiotics.

\section{Measurement of ATP formation}

Tibialis anterior and diaphragm muscles were dissected, trimmed clean of visible fat and connective tissue, minced with scissors and digested in ATP medium, containing $50 \mathrm{mM}$ Tris- $\mathrm{HCl}$ (pH 7.4), $100 \mathrm{mM} \mathrm{KCl}, 5 \mathrm{mM}$ $\mathrm{MgCl}_{2}, 1.8 \mathrm{mM}$ ATP, $1 \mathrm{mM}$ ethylenediaminetetraacetic acid (EDTA), and $0.1 \%$ collagenase type $\mathrm{V}$ for 10 minutes at $37^{\circ} \mathrm{C}$ under strong agitation. After centrifugation, the pellet was homogenised with Ultra-Turrax T10 (Ika-lab, Staufen, Germany) for 10 seconds at maximum speed in ATP medium. The mitochondrial fraction, obtained by different centrifugations $(380 \mathrm{~g}$ and $10,000 \mathrm{~g}$ for five minutes at $4^{\circ} \mathrm{C}$ ), was then suspended in a mitochondria resuspension buffer containing $12.5 \mathrm{mM}$ Tris acetate,

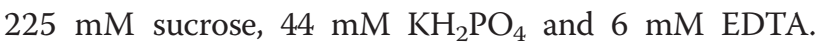
Total oxidative phosphorylation (OXPHOS)-ATP in isolated mitochondria was measured by the luciferin-luciferase method, as described, with slight modifications [25]. Briefly, mitochondria were plated in 96 wells and treated with buffer-A $(150 \mathrm{mM} \mathrm{KCl}, 25 \mathrm{mM}$ Tris- $\mathrm{HCl}, 2 \mathrm{mM}$ EDTA,

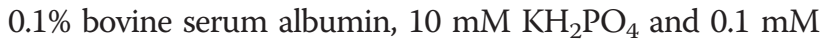
$\mathrm{MgCl}_{2}$ (pH 7.4) containing $0.8 \mathrm{M}$ malate, $2 \mathrm{M}$ glutamate, $500 \mathrm{mM}$ ADP, $100 \mathrm{mM}$ luciferin and $1 \mathrm{mg} / \mathrm{ml}$ luciferase. Oligomycin $(2 \mu \mathrm{g} / \mathrm{ml})$ was also used to detect the presence of glycolytic ATP. OXPHOS-ATP was measured using a GloMax luminometer (Promega, Milan, Italy).

\section{High-resolution respirometry}

Respiratory chain defects were assessed in tibialis anterior and diaphragm fibre bundles using published protocols [40-42]. After transferring the tissue sample into ice-cold BIOPS (10 $\mathrm{mM} \mathrm{CaK}_{2}$ ethyleneglycoltetraacetic acid (EGTA) buffer, $7.23 \mathrm{mM} \mathrm{K} \mathrm{K}_{2}$ EGTA buffer, $0.1 \mu \mathrm{M}$ free calcium, $20 \mathrm{mM}$ imidazole, $20 \mathrm{mM}$ taurine, $50 \mathrm{mM}$ 2-(N-morpholino)ethanesulfonic acid hydrate, $0.5 \mathrm{mM}$ dithiothreitol, $6.5 \mathrm{mM} \mathrm{MgCl} 26 \mathrm{H}_{2} \mathrm{O}, 5.7 \mathrm{mM}$ ATP and $15 \mathrm{mM}$ phosphocreatine ( $\mathrm{pH}$ 7.1)), connective tissue was removed and the muscle fibres were mechanically separated. Complete permeabilisation of the plasma membrane was ensured by gentle agitation for 30 minutes at $4^{\circ} \mathrm{C}$ in $2 \mathrm{ml}$ of BIOPS solution containing $50 \mu \mathrm{g} / \mathrm{ml}$ saponin. The fibre bundles were rinsed by agitation for 10 minutes in ice-cold mitochondrial respiration medium (MiR05; $0.5 \mathrm{mM}$ EGTA, $3 \mathrm{mM} \mathrm{MgCl}$,

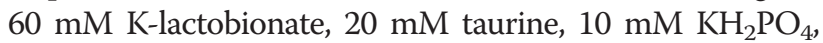
$20 \mathrm{mM}$ Hepes, $110 \mathrm{mM}$ sucrose and $1 \mathrm{~g} / \mathrm{l}$ bovine serum albumin ( $\mathrm{pH}$ 7.1). The permeabilised muscle fibres were weighed and added to an Oxygraph-2 k respiratory chamber (Oroboros Instruments, Innsbruck, Austria) containing $2 \mathrm{ml}$ of MiR06 (MiR05 supplemented with $280 \mathrm{U} / \mathrm{ml}$ catalase at $37^{\circ} \mathrm{C}$ ). Oxygen flux per muscle mass was recorded online using DatLab software (Oroboros Instruments). After calibration of the oxygen sensors at air saturation, a few $\mu$ of $\mathrm{H}_{2} \mathrm{O}_{2}$ were injected into the chamber to reach a concentration of $400 \mu \mathrm{M} \mathrm{O}_{2}$. In order to detect the electron flow through CI and CII mitochondrial complexes, titrations of all of substrates, uncouplers and inhibitors were added in series as previously described $[41,42]$. The measurement of CIV respiration was obtained by addition of the artificial substrates N,N,N,N'N'-tetramethyl-p-phenylenediamine dihydrochloride and ascorbate [40]. Oxygen fluxes were corrected by subtracting residual oxygen consumption from each measured mitochondrial steady-state. Respirometry measurements were performed in duplicate on each specimen.

\section{Real-time quantitative PCR}

Satellite cells and muscle tissue samples were homogenised, and RNA was extracted using the TRIzol protocol 
(Invitrogen-Life Technologies, Monza, Italy). Using published protocols [43], after solubilisation in RNase-free water, first-strand cDNA was generated from $1 \mu \mathrm{g}$ of total RNA using the ImProm-II Reverse Transcription System (Promega). As show in Table 1, a set of primer pairs amplifying fragments ranging from 85 to 247 bp was designed to hybridise to unique regions of the appropriate gene sequence. Real-time quantitative PCR (qPCR) was performed using the SYBR Green Supermix (Bio-Rad, Hercules, CA, USA) on a Roche LightCycler 480 Instrument (Roche, Basel, Switzerland). All reactions were run in triplicate. A melt-curve analysis was performed at the end of each experiment to verify that a single product per primer pair was amplified. As a control experiment, gel electrophoresis was performed to verify the specificity and size of the amplified qPCR products. Samples were analysed using the Roche LightCycler 480 software and the second derivative maximum method. The fold increase or decrease was determined relative to a calibrator after normalising to 36b4 (internal standard) through the use of the formula $2^{-\Delta \Delta C T}$ [44].

Mitochondrial DNA (mtDNA) from muscle tissue samples was quantified as described with slight modifications [45]. Briefly, total DNA was extracted with the QIAamp DNA mini kit (Qiagen, Milano, Italy). Twenty ng of total DNA was assessed by qPCR. RNaseP gene was used as an endogenous control for nuclear DNA and the cytochrome $b$ gene as a marker for mtDNA. Primer sequences are shown in Table 1.
In vivo imaging using two-photon confocal microscopy Mitochondrial morphology and autophagosome formation in living animals were monitored in tibialis anterior muscles transfected by electroporation with plasmids encoding pDsRed2-Mito or the LC3 protein fused to the yellow fluorescent protein (YFP-LC3), as described previously $[29,38,46]$. Two-photon confocal microscopy in the live, anaesthetised animals was then performed 12 days later on in situ exposure of transfected muscles $[29,38,46]$. To allow the muscle to recover from the injection-induced swelling, microscopic observation was interrupted for two to five minutes.

\section{Transmission electron microscopy}

Tibialis anterior muscles were dissected and fixed for one hour in a solution containing $4 \%$ paraformaldehyde and $0.5 \%$ glutaraldehyde in $0.1 \mathrm{M}$ cacodylate buffer, $\mathrm{pH}$ 7.4, immobilised on a Nunc Sylgard coated Petri dish (ThermoFisher Scientific, Waltham, MA, USA) to prevent muscular contraction as previously described [47]. The muscles were rinsed in the same buffer and dissected further into small blocks that were subsequently processed for transmission electron microscopy (TEM) as described elsewhere [48]. Briefly, the samples were postfixed with osmium tetroxide $(2 \%$ in cacodylate buffer), rinsed, en bloc stained with $1 \%$ uranyl acetate in $20 \%$ ethanol, dehydrated and embedded in epoxy resin (Epon 812; Electron Microscopy Science, Hatfield, PA, USA) that was baked for 48 hours at $67^{\circ} \mathrm{C}$. Thin sections

Table 1 Primer pairs designed for qPCR analysis

\begin{tabular}{|c|c|c|c|}
\hline Name/symbol & Gene accession Number & Primer sequence & Amplicon \\
\hline \multirow[t]{2}{*}{ Atg $4 b$} & NM_174874 & F: 5'-ATTGCTGTGGGGTTITTCTG-3' & $247 \mathrm{bp}$ \\
\hline & & R: 5'-AACCCCAGGATTTTCAGAGG-3' & \\
\hline \multirow[t]{2}{*}{ Atrogin-1 (fbxo32) } & NM_026346 & F: 5'-GCAAACACTGCCACATTCTCTC-3' & $93 \mathrm{bp}$ \\
\hline & & R: 5'-CTTGAGGGGAAAGTGAGACG-3' & \\
\hline \multirow[t]{2}{*}{ Bnip3 } & NM_009760 & F: 5'-TTCCACTAGCACCTTCTGATGA-3' & $150 \mathrm{bp}$ \\
\hline & & 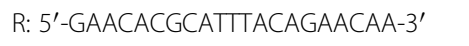 & \\
\hline \multirow[t]{2}{*}{ Cytochrome b (mt-cytb) } & NC_005089 & F: 5'-ACGCCATTCTACGCTCTATC-3' & $95 \mathrm{bp}$ \\
\hline & & R: 5'-GCTTCGTTGCTTTGAGGTGT-3' & \\
\hline \multirow[t]{2}{*}{ MuRF1 (Trim63) } & NM_001039048 & F: 5'-ACCTGCTGGTGGAAAACATC-3' & $96 \mathrm{bp}$ \\
\hline & & R: 5'-CTTCGTGTTCCTTGCACATC-3' & \\
\hline \multirow[t]{2}{*}{ MUSA1 (fbxo30) } & NM_001168297, NM_027968 & F: 5'-TCGTGGAATGGTAATCTTGC-3' & $191 \mathrm{bp}$ \\
\hline & & R: 5'-CCTCCCGTTTCTCTATCACG-3' & \\
\hline \multirow[t]{2}{*}{ p62 (Sqstm1) } & NM_011018 & F: 5'-GAAGCTGCCCTATACCCACA-3' & $85 \mathrm{bp}$ \\
\hline & & R: 5'-AGAAACCCATGGACAGCATC-3' & \\
\hline \multirow[t]{2}{*}{ RNaseP (Rpp30) } & NM_019428 & F: 5'-GAAGGCTCTGCGCGGACTCG-3' & $100 \mathrm{bp}$ \\
\hline & & R: 5'-CGAGAGACCGGAATGGGGCCT-3' & \\
\hline \multirow[t]{2}{*}{3664 (Rplpo) } & NM_007475 & F: 5'-AGGATATGGGATTCGGTCTCTTC-3' & $143 \mathrm{bp}$ \\
\hline & & R: 5'-TCATCCTGCTTAAGTGAACAAACT-3' & \\
\hline
\end{tabular}

F: forward, R: reverse. 
were obtained with a Leica ultramicrotome (Reichert Ultracut E and UC7; Leica Microsystems, Wetzlar, Germany) stained with uranyl acetate and lead citrate, and finally examined with a Philips CM10 TEM (Philips, Eindhoven, The Netherlands). Morphometric analysis of mitochondrial cristae complexity was evaluated with a stereological method. Briefly, a regular grid has been superimposed over 10500X TEM micrographs and the number of intersections between the grid and mitochondrial cristae was recorded. The same grid was used for all the different analysis.

\section{Protein isolation and western blotting}

Satellite cells were harvested and homogenised for 10 minutes at $4^{\circ} \mathrm{C}$ in RIPA lysis buffer, containing $50 \mathrm{mM}$ Tris- $\mathrm{HCl}$ (pH 7.4), $150 \mathrm{mM} \mathrm{NaCl}, 1 \% \mathrm{NP}-40,1 \%$ sodium deoxycholate, $1 \mathrm{mM}$ EDTA and $0.1 \%$ sodium dodecyl sulphate (SDS). Tissue samples from muscles were homogenised in a lysis buffer containing $20 \mathrm{mM}$ Tris- $\mathrm{HCl}$ (pH 7.4), $150 \mathrm{mM} \mathrm{NaCl}, 1 \%$ Triton X-100, 10\% glycerol, $10 \mathrm{mM}$ EGTA and 2\% SDS. Buffers were supplemented with a cocktail of protease and phosphatase inhibitors (cOmplete and PhosSTOP; Roche). Protein concentration was determined using the bicinchoninic acid assay (ThermoFisher Scientific). Using published protocols [49], SDS and $\beta$-mercaptoethanol were added to samples before boiling, and equal amounts of proteins (40 $\mu \mathrm{g} / \mathrm{lane})$ were separated by $4 \%$ to $20 \%$ SDS-polyacrylamide gel electrophoresis (Criterion TGX Stain-free precast gels and Criterion Cell system; Bio-Rad). Proteins were then transferred onto a nitrocellulose membrane using a Bio-Rad Trans-Blot Turbo System. The membranes were probed using the following primary antibodies as indicated in the text: goat polyclonal anti-HSP60 (N-20) and rabbit polyclonal anti-MyoD (C-20) (Santa Cruz Biotechnology, Dallas, TX, USA), mouse monoclonal anti-ClpP and rabbit polyclonal anti-LC3B (Sigma-Aldrich, Saint Louis, MO, USA), rabbit polyclonal anti-Mul-1 (Abcam, Cambridge, UK), mouse monoclonal anti-sarcomeric myosin (MF20) (Developmental Studies Hybridoma Bank, Iowa City, IA, USA), rabbit polyclonal anti-phospho-FoxO3a (Ser253), rabbit polyclonal anti-phospho-S6 ribosomal protein (Ser240/244), rabbit monoclonal anti-phospho-4E-BP1 (Thr37/46) (263B4) and rabbit polyclonal anti-phosphoAkt (Ser473) (Cell Signaling Technology, Danvers, MA, USA). After the incubation with the appropriate horseradish-peroxidase-conjugated secondary antibody (Cell Signaling Technology), bands were visualised using the Bio-Rad Clarity Western ECL substrate with a Bio-Rad ChemiDoc MP imaging system. To monitor for potential artefacts in loading and transfer among samples in different lanes, the blots were routinely treated with the Restore Western Blot Stripping Buffer (ThermoFisher Scientific) and reprobed with rabbit polyclonal anti-calnexin
(GeneTex, Irvine, CA, USA), goat polyclonal anti-actin (I-19) or rabbit polyclonal anti-GAPDH (FL-335) primary antibodies (Santa Cruz Biotechnology). When appropriate, rabbit polyclonal anti-FoxO3a (75D8), rabbit monoclonal S6 ribosomal protein (54D2), rabbit polyclonal 4E-BP1 (53H11), and rabbit polyclonal Akt primary antibodies (Cell Signaling Technology) that recognise the protein independently of its phosphorylation state were also used in reprobing experiments.

\section{Confocal microscopy of myogenic precursor cells}

Cells were plated in eight-well Nunc LabTeck Chamber slides (ThermoFisher Scientific). When indicated cells were transfected with YFP-LC3 plasmid. Transfections were performed with the Lipofectamine LTX with Plus reagent (Invitrogen-Life Technologies) according to the manufacturer's instructions. The cells were used 24 hours after transfection in the various experimental settings described. For confocal imaging, the cells were fixed in paraformaldehyde and washed in phosphate-buffered saline [50]. To prevent nonspecific background, cells were incubated in $10 \%$ goat serum/phosphate-buffered saline followed by probing with the primary antibody mouse monoclonal anti-cyclophillin D (Abcam). Cells were then incubated with the secondary antibody, Alexa Fluor 546 dye-conjugated anti-mouse IgG (Molecular Probes-Life Technologies, Monza, Italy). Slides were placed on the stage of a TCS SP2 Laser-Scanning Confocal microscope (Leica Microsystems) equipped with an electronically controlled and freely definable Acousto-Optical Beam Splitter. Images were acquired with x63 magnification oil-immersion lenses. Analyses were performed using Imagetool software (Health Science Center, University of Texas, San Antonio, TX, USA). Images of cells expressing YFP-LC3 were thresholded by using the automatic threshold function.

\section{Immunohistochemistry and histology}

Laminin and haematoxylin and eosin ( $\mathrm{H}$ \& $\mathrm{E})$ staining were performed as previously described $[47,51]$. To measure the cross sectional area (CSA) of myofibres, muscle sections were stained with an anti-laminin A antibody (L1293; Sigma-Aldrich). Laminin, a cell-adhesion molecule strongly expressed in the basement membrane of skeletal muscle, was detected using an appropriate secondary antibody. Morphometric analyses were performed on sections collected from similar regions of each muscle using a Leica DMI4000 B automated inverted microscope equipped with a DCF310 digital camera. Image acquisition was controlled by the Leica LAS AF software. The ImageJ software was used to determine the CSA of 1,000 to 3,000 individual fibres from at least two different fields for each muscle section. Four to nine sections from each muscle were analysed. For histological analyses, serial muscle 
sections were obtained and stained in $\mathrm{H}$ \& $\mathrm{E}$ following standard procedures. The number of fibres was counted and analysed using the ImageJ software.

Single myofiber isolation of hind limb muscle and nuclei immunofluorescence on single fibers was performed as previously described [8]. Nuclei of 30 individual fibres from each muscle were analysed.

\section{Whole body tension}

The whole body tension (WBT) procedure was used to determine the ability of mice to exert tension in a forward pulling manoeuvre that is elicited by stroking the tail of the mice [52]. The tails were connected to a Grass FT03 transducer (Astro-Med, West Warwick, RI, USA) with a 4.0 silk thread (one end of the thread being tied to the tail and the other end to the transducer) [47]. Each mouse was placed into a small tube constructed of a metal screen with a grid spacing of $2 \mathrm{~mm}$. The mice entered the apparatus and exerted a small resting tension on the transducer. Forward pulling movements were elicited by a standardised stroke of the tail with serrated forceps, and the corresponding forward pulling tensions were recorded using a Grass Polyview recording system (Astro-Med). Between 20 and 30 strokes of the tail forward pulling tensions were generally recorded during each session. The WBT was determined by dividing the average of the top ten or top five forward pulling tensions, respectively, by the body weight and represent the maximum phasic tension that can be developed over several attempts [52]. It is important to note that treatments or conditions which primarily alter muscle mass without changing the tension developed per unit of muscle mass produce corresponding alterations in forward pulling tension that are not associated with changes in either WBT 5 or WBT $10[52,53]$.

\section{Treadmill running}

Animals were made to run on a standard treadmill machine (Columbus Instruments, Columbus, OH, USA) either on a $0 \%$ grade or tilted $10 \%$ downhill starting at a warm-up speed of $5 \mathrm{~m} /$ minute for five minutes [54]. Every subsequent five minutes, the speed was increased by $5 \mathrm{~m} /$ minute until the mice were exhausted. Exhaustion was defined as the inability of the animal to return to running within 10 seconds after direct contact on an electric stimulus grid. Running time was measured and running distance calculated. Distance is the product of time and speed of the treadmill.

As a measure of membrane permeability, the Evans blue dye (EBD) assay was used [47]. A concentration of $5 \mu \mathrm{g} / \mu \mathrm{l}$ EBD prepared in physiological saline was injected intravenously through the tail vein. Injections $(50 \mu \mathrm{l} / 10 \mathrm{~g}$ body weight) were performed 20 to 30 minutes after treadmill running. Mice were sacrificed 24 hours after EBD injection. tibialis anterior muscle sections (20 to 30 from each muscle) were then collected and the immunofluorescence of EBD-positive fibres was imaged using Texas red red filter. Creatine kinase (CK) serum levels (units per litre) were measured in blood samples obtained from the tail vein of mice after treadmill running. The blood was centrifuged at $13,000 \times g$ at $4^{\circ} \mathrm{C}$ and the supernatant used to measure CK activity in an indirect colorimetric assay (Randox Laboratories, Crumlin, Northern Ireland, UK) $[16,18]$.

\section{Statistics}

Upon verification of normal distribution, the statistical significance of the raw data between the groups in each experiment was evaluated using the unpaired Student's $t$-test (single comparisons) or one way analysis of variance (ANOVA) followed by the Newman-Keuls post-test (multiple comparisons). The GraphPad Prism software package (GraphPad Software, La Jolla, CA, USA) was used. After statistics (raw data), data from different experiments were represented and averaged in the same graph. The results are expressed as means \pm SEM of the indicated $\mathrm{n}$ values.

\section{Chemicals}

pDsRed2-Mito was a gift of Prof. Luca Scorrano (University of Padova, Padova, Italy). Dispase was purchased from Gibco-Life Technologies (Monza, Italy). TMRM and the secondary antibody for laminin experiments were obtained from Molecular Probes-Life Technologies. Iscove's modified Dulbecco's medium, penicillin, streptomycin, gentamycin, horse serum, and foetal bovine serum were purchased from Euroclone (Pero, Italy). Matrigel was obtained from BDBioscience (Milano, Italy). Primer pairs were obtained from Primmbiotech (Milano, Italy). Fibroblast growth factor was purchased from Tebu-bio (Milano, Italy). DETA-NO and KT5823 were obtained from Merck Millipore (Darmstadt, Germany). ODQ and cyclosporine were purchased from Enzo Life Sciences (Farmingdale, NY, USA). L ${ }^{\omega}$-arginine methylester (L-NAME) and the other chemicals were purchased from Sigma-Aldrich.

\section{Results}

\section{nNOS $\mu$ deficiency leads to mitochondrial dysfunction}

Mitochondrial function in skeletal muscles of adult NOS1-/- mice, that is, at P120, was dissected and compared with that of the respective age-matched wild-type littermattes (control). Mitochondrial membrane potential was monitored in isolated fibres from flexor digitorum brevis muscles loaded with TMRM, a potentiometric fluorescent dye. TMRM accumulates in the mitochondria that maintain a polarised mitochondrial membrane potential. A latent mitochondrial dysfunction masked by the ATP synthase operating in a reverse mode, that is, to consume ATP in order to maintain the mitochondrial membrane 
potential, can be unveiled using the ATP synthase inhibitor oligomycin [25]. In agreement with previous reports [29], addition of oligomycin to control mice fibres did not cause immediate changes in membrane potential even after extensive incubation (Figure 1A). Conversely, mitochondria in fibres of NOS1-/- mice underwent marked depolarisation after oligomycin.

We investigated whether the latent mitochondrial dysfunction observed in muscles of NOS1-/- mice affected the muscle bioenergetic parameters. To this end, we measured ATP generation from OXPHOS in isolated mitochondria of tibialis anterior and diaphragm muscle fibres. As shown in Figure 1B, total OXPHOS-generated ATP was significantly lower in NOS1-/- mice when compared to control.

We then analysed the mitochondrial bioenergetics in intact fibres using an in situ approach measuring oxygen consumption by high resolution respirometry. By this

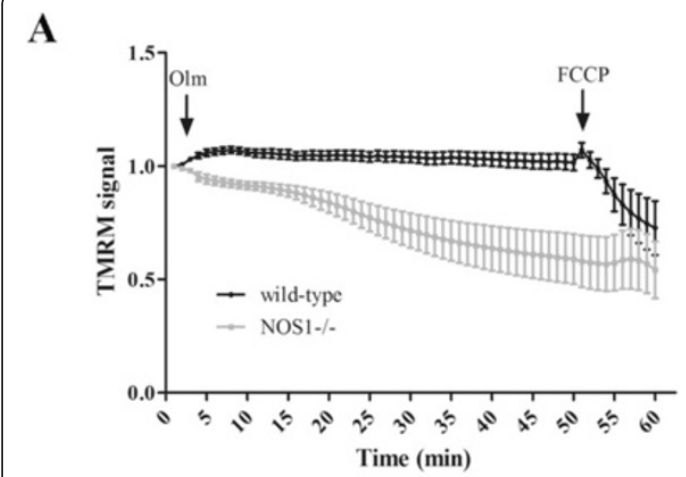

C CI
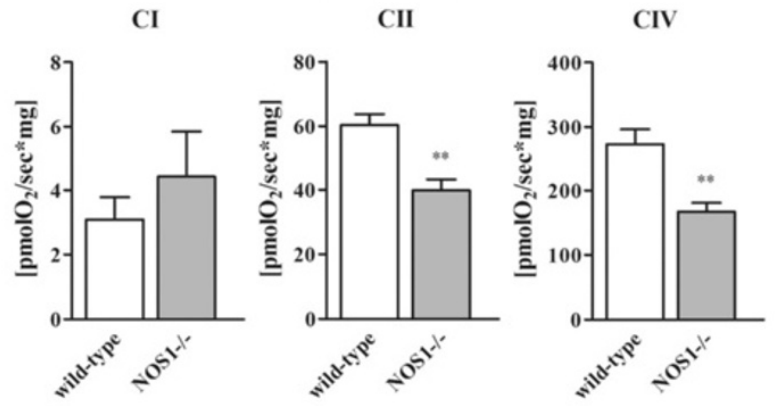

E

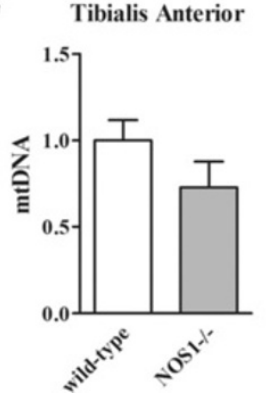

Tibialis Anterior

CII
B
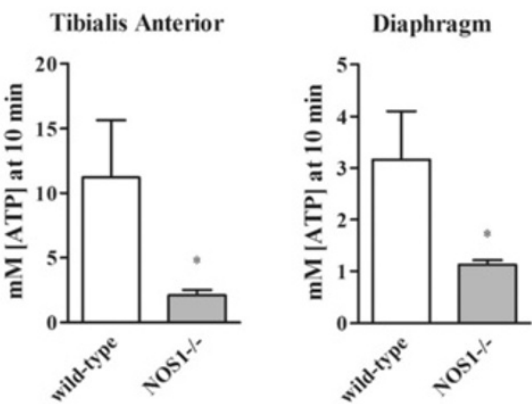

D

CI
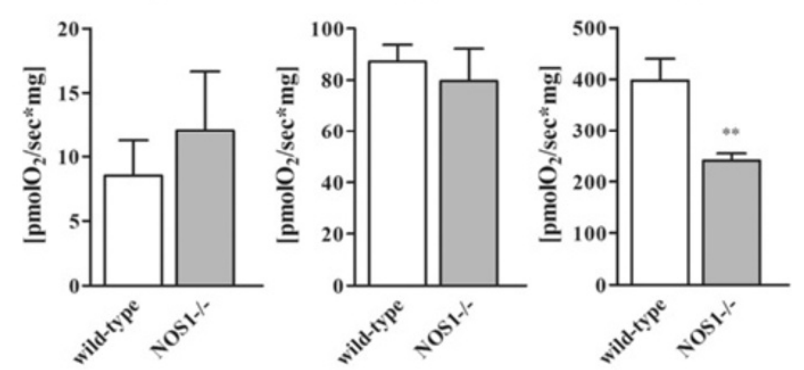

Figure 1 Mitochondrial metabolism is impaired in skeletal muscles of NOS1-/- mice. Fibres were isolated from different muscles of wild-type and NOS1-/- mice at P120. (A) Mitochondrial membrane potential measured in fibres isolated from flexor digitorum brevis muscles, loaded with TMRM and treated with $5 \mu \mathrm{M}$ oligomycin (Olm) or $4 \mu \mathrm{M}$ FCCP. TMRM staining was monitored in six to ten fibres obtained from at least three different animals per experimental group. Data are expressed by setting the initial value as 1. (B) ATP production on mitochondria isolated from tibialis anterior and diaphragm muscles, at 10 minutes after substrate addition. Data are expressed by setting the initial value as 1. (C-D) Oxygen consumption on fibres isolated from tibialis anterior and diaphragm muscles, supplied with specific $\mathrm{Cl}, \mathrm{Cll}$ and $\mathrm{CIV}$ mitochondrial complex substrates, as indicated in the Methods. (E) Quantitative analysis of the mtDNA copy number. Data are expressed by normalizing mtDNA values versus nuclear DNA. Each histogram represents the data obtained from at least five different animals per experimental group. ${ }^{*} P<0.05$ and ${ }^{* *} P<0.01$ versus the respective wild-type control. 
approach, we found that the maximal tissue massspecific OXPHOS capacity with physiological combinations of CI mitochondrial complex substrates was similar in both tibialis anterior and diaphragm of NOS1-/- and control mice (Figure 1C-D). In contrast, the CII-linked respiratory capacity in tibialis anterior of NOS1-/- mice was lower than that in control muscle fibres, while no difference was observed in the diaphragm. In both tibialis anterior and diaphragm of NOS1-/- mice the CIVlinked respiratory capacity decreased significantly with respect to the controls. Of interest, qPCR analysis of mtDNA levels in tibialis anterior and diaphragm muscles did not reveal any difference between NOS1-/- and control mice (Figure 1E) suggesting that mitochondrial mass was not affected and defects in OXPHOS were due to dysfunctional mitochondria.

\section{$\mathrm{nNOS} \mu$ deficiency affects mitochondrial network remodelling, UPR ${ }^{\mathrm{mt}}$ and autophagy}

Alterations in the content, shape or function of the mitochondria have been associated with muscle homeostasis $[31,55]$. To identify the changes in mitochondrial network morphology, tibialis anterior muscles of P120 NOS1-/- and wild-type control mice were imaged using pDsRed2-Mito, a mitochondrially targeted red fluorescent protein, by in situ two-photon confocal microscopy $[29,38,46]$. NOS1-/- mice showed a disorganised mitochondrial network (Figure 2A). Accordingly, ultrastructural analyses by TEM (Figure $2 \mathrm{~B}$ and Additional file 1: Figure S1A) revealed changes in the subsarcolemmal mitochondria of tibialis anterior muscles of NOS1-/- mice that exhibited, in thin sections, a significant increase in mitochondrial surface area (Figure 2C) and a significant decrease in the density of the cristae (Figure 2D), as compared with the controls. The same evaluation was performed on subsarcolemmal mitochondria from diaphragm muscle with similar results (data not shown). The analysis of intermyofibrillar mitochondria (see Additional file 1: Figure S1B) showed a pattern of enlarged mitochondria indicating that the presence of these mitochondrial alterations in NOS1-/- mice muscle is not restricted to the sarcolemma but is a more general phenomenon.

We then analysed two downstream processes linked to mitochondrial stress: UPR ${ }^{\mathrm{mt}}$ and autophagy. In tibialis anterior muscles of P120 NOS1-/- mice the expression of the nuclearly-encoded mitochondrial chaperones HSP60 and the protease ClpP, which correlates with the level of unfolded proteins in mitochondria [56,57], was found to be higher than in the controls (Figure 2E). In addition, the two-photon confocal microscopy of the YFP-LC3 $[29,38,46]$ revealed the presence of LC3positive vesicles, an established marker of autophagosome formation [58], in tibialis anterior muscles of P120 NOS1-/- mice (Figure 2F). Furthermore, TEM analysis showed the presence of autophagic vacuoles and multivesicular bodies, indicative of an active autophagic pathway [59], in tibialis anterior and diaphragm muscles of P120 NOS1-/- mice (Figure 2B and Additional file 1: Figure S1C). The enhanced autophagy in the absence of $n N O S \mu$ in skeletal muscle was confirmed by Western blot analysis. The appearance of a faster migrating band of LC3 protein due to its lipidation and cleavage is a common marker of autophagy induction [58]. As shown in Figure 2G, tibialis anterior muscles of P120 NOS1-/mice exhibited increased lipidated LC3 levels when compared to control mice. Similar results on LC3 conversion were obtained analysing diaphragm muscle samples (see Additional file 1: Figure S1D).

NO signalling regulates UPR ${ }^{\mathrm{mt}}$ and autophagy machinery Activation of the NO-dependent enzyme guanylate cyclase, with formation of cyclic guanosine monophosphate (cGMP) and activation of a variety of downstream signalling cascades, including cGMP-dependent-protein kinases (PKG), contributes significantly to mediate the physiological effects of NO in muscle $[2,5,60]$. To investigate the involvement of the cGMP-dependent signalling on $\mathrm{UPR}^{\mathrm{mt}}$ and autophagy, myogenic precursor cells were differentiated for six hours in the absence (control) or in the presence of the inhibitor of NOS L-NAME (6 mM), the inhibitor of guanylate cyclase ODQ $(10 \mu \mathrm{M})$, and the inhibitor of PKG KT5823 (1 $\mu \mathrm{M})$ [61-66]. L-NAME, ODQ and KT5823 treatment increased the expression of HSP60 and ClpP protein. The NO donor DETA-NO $(80 \mu \mathrm{M})$ and the membrane-permeant cGMP analogue $8 \mathrm{Br}$-cGMP (2.5 mM) [62-66] reversed the effects of L-NAME and ODQ, respectively (Figure $3 \mathrm{~A}$ ).

In another set of experiments, cells were transiently transfected with YFP-LC3 and then differentiated. As shown by confocal microscopy fluorescence analysis of LC3 and the mitochondrial matrix-specific protein cyclophillin D (Figure 3B), in control cells LC3 staining was diffuse and the majority of mitochondria were in the elongated form, indicating myogenic differentiation [25] and a low rate of autophagy. L-NAME, ODQ, and KT5823 treatment, while inducing mitochondrial fragmentation, resulted in LC3 localisation into dot cytoplasmic structures, as compared to the diffuse cytoplasmic distribution observed in control cells. The effects of L-NAME and ODQ were prevented by DETA-NO and 8Br-cGMP, respectively.

NO control of autophagy was assessed further by analysing the expression of relevant markers of the autophagic signalling pathway, namely LC3, by western blotting and p62, Bnip3 and Atg4 by qPCR analysis [58,67]. L-NAME, ODQ and KT5823 treatments increased lipidated LC3 conversion in differentiated satellite cells and LC3 lipidation induced by L-NAME and ODQ was blocked by 


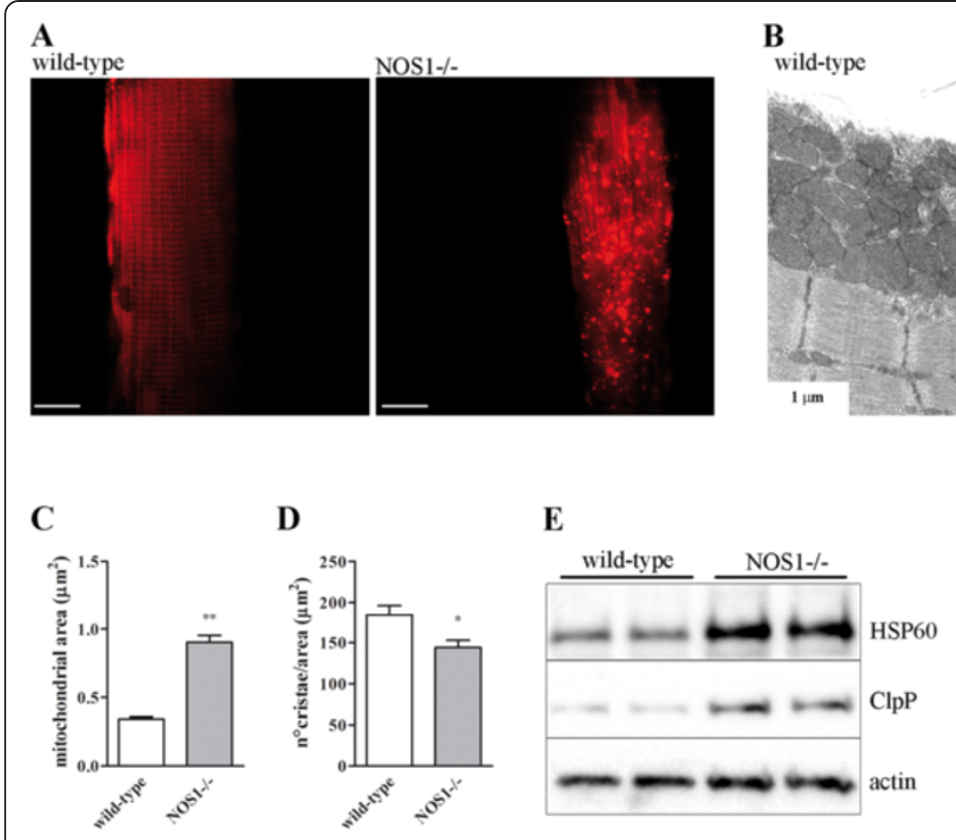

G
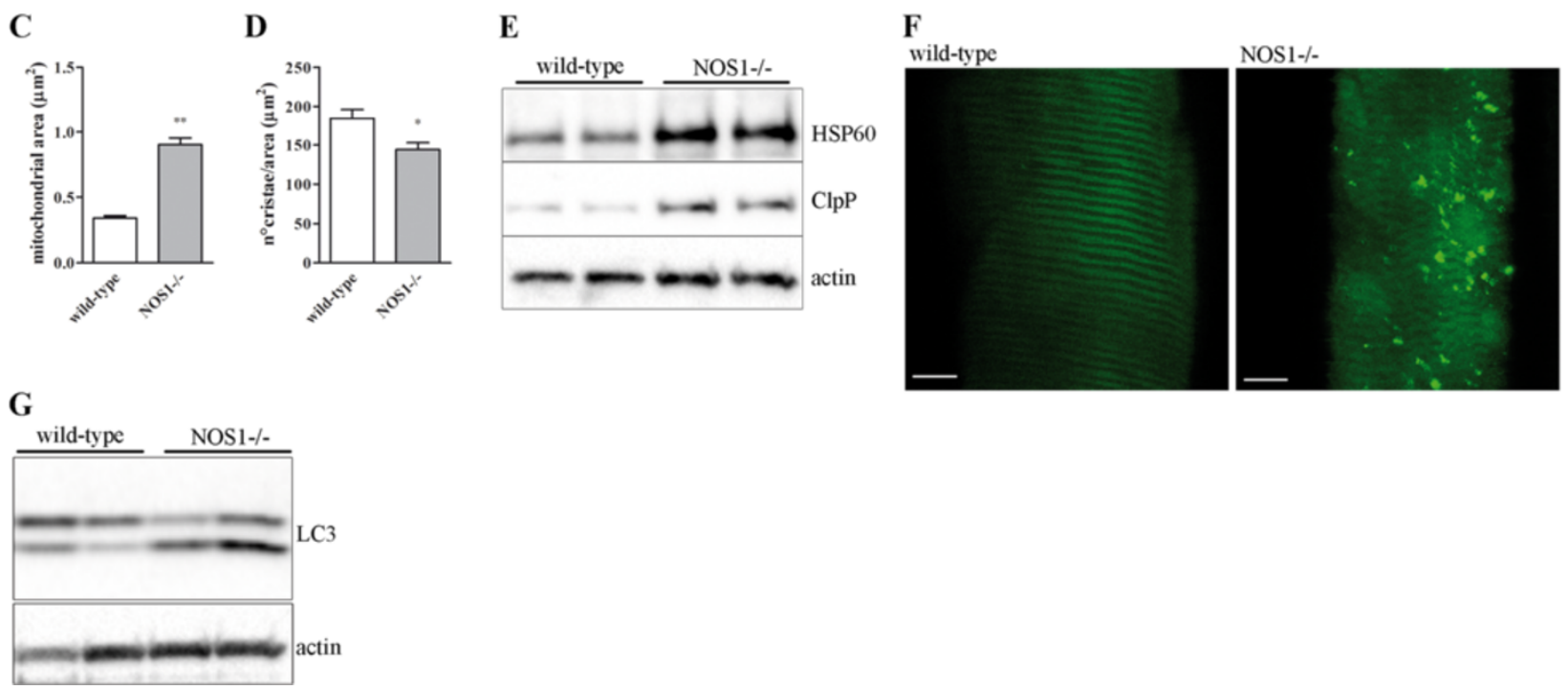

Figure 2 Mitochondrial morphology, UPR ${ }^{\mathrm{mt}}$ and autophagy in skeletal muscles of NOS1-/- mice. Tibialis anterior muscles were isolated from wild-type and NOS1-/- mice at P120. (A) In vivo imaging of the mitochondrial network by two-photon confocal microscopy. Muscles were transfected with the mitochondrially targeted red fluorescent protein pDsRed2-Mito. The images are representative of results obtained from at least five different animals per experimental group. Scale bar: $10 \mu \mathrm{m}$. (B) TEM images detecting the presence of abnormal, enlarged subsarcolemmal mitochondria (asterisks) or autophagic vacuoles (arrowheads) in NOS1-/- muscles. The inset depicts a multivesicular body in NOS1-/- fibres taken at higher magnification. The images are representative of results obtained from at least three different animals per experimental group. (C-D) Subsarcolemmal mitochondrial ultrastructure analysis by TEM. Data represent the quantification of the mitochondrial area and morphometric analysis of mitochondrial cristae complexity. Each histogram represents the data obtained from at least three different animals per experimental group. ${ }^{*} P<0.05$ and ${ }^{* *} P<0.01$ versus the respective wild-type control. (E) Western blot analysis of HSP60 and ClpP expression. Actin was used as the internal standard. The image is representative of results obtained from at least five to seven different animals per experimental group. (F) In vivo imaging of autophagosome formation by two-photon confocal microscopy. Muscles were transfected with YFP-LC3. The images are representative of results obtained from at least five different animals per experimental group. Scale bar: $10 \mu \mathrm{m}$. (G) Western blot analysis of LC3 lipidation. Actin was used as the internal standard. The image is representative of results obtained from at least 10 different animals per experimental group.

DETA-NO or $8 \mathrm{Br}-\mathrm{cGMP}$, respectively (Figure $4 \mathrm{~A}$ ). In addition, cells treated with L-NAME, ODQ and KT5823 expressed higher levels of transcripts encoding p62, Bnip3 and Atg4 (Figure 4B).

\section{Deficient nitric oxide signalling promotes FoxO3-Mul-1 axis}

Catabolic conditions activate FoxO transcription factors, which stimulate the ubiquitin-proteasome system as a response to skeletal muscle-wasting $[31,55]$. FoxO3 activity is necessary and sufficient for the induction of autophagy in skeletal muscle [38]. FoxO3 translocation from the cytoplasm to the nucleus determines the direct transcriptional activation of genes essential to autophagosome formation, namely p62, Bnip3 and Atg4 [58,67]. Enhanced activity of FoxO transcription factors has also been associated with disruption of mitochondrial function and organisation leading to impaired skeletal muscle function and development [29]. As shown in Figure 5A, C, phosphorylated FoxO3 levels in tibialis anterior and diaphragm muscles of P120 NOS1-/- mice were lower than in the controls. In addition, tibialis anterior and diaphragm muscles of NOS1-/- mice overexpressed the protein corresponding to mitochondrial 


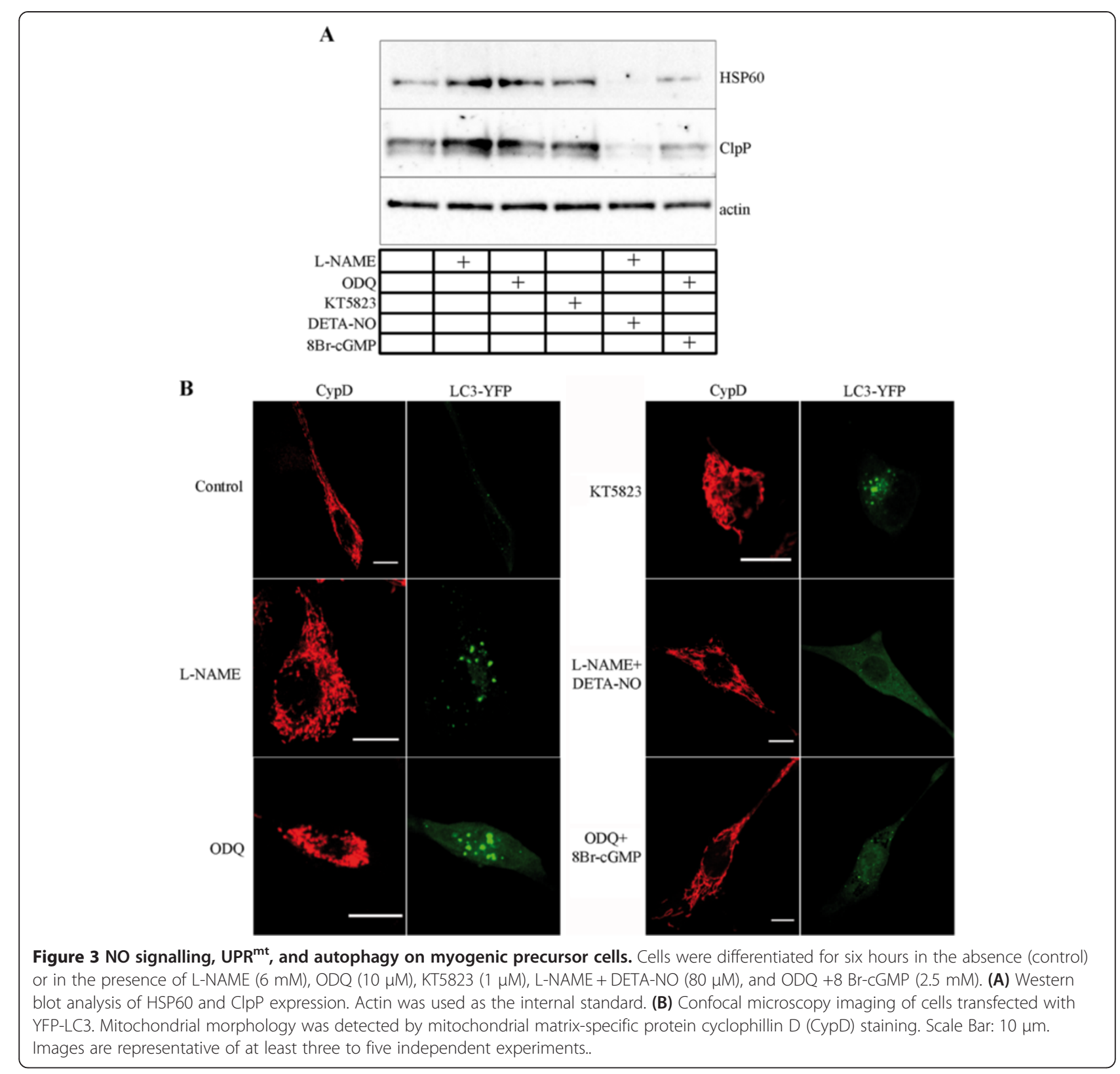

ubiquitin ligase Mul-1 (Figure 5B, D), which has been recently reported to be upregulated in muscle through FoxO3 transcription factors and promoting mitochondrial fission, depolarization and mitophagy [68,69]. As shown in Figure $5 \mathrm{E}$, in vitro treatment of differentiated myogenic precursor cells from wild-type control mice with L-NAME, ODQ and KT5823 increased Mul-1 protein expression. The effects induced by L-NAME and ODQ were blocked by DETA-NO and 8Br-cGMP, respectively. In tibialis anterior and diaphragm muscles of P120 NOS1-/- mice, qPCR analysis of other E3 ubiquitin ligases, atrogin-1 and MuRF1, involved in muscle loss $[69,70]$, ruled out a nNOS $\mu$-dependent modulation of their expression (Figure 5F, G). Also, the differences obtained with MUSA1 analysis are difficult to correlate with nNOS $\mu$ deficiency. These findings indicate that the effects of nNOS $\mu$ absence on E3 ubiquitin ligases mainly affect expression of Mul-1 gene.

\section{nNOS $\mu$ deficiency affects muscle growth}

We evaluated the effects of the absence of nNOS $\mu$ on skeletal muscle phenotype. Tibialis anterior, gastrocnemius, soleus, and extensor digitorum longus muscles were dissected and weighed. Since the body weight and the visceral adipose tissue of NOS1-/- male mice were significantly lower than wild-type control (see Additional file 2: Figure S2A-B) [71] we calculated the muscle size relative to body weight [72]. As shown in Figure 6A and 


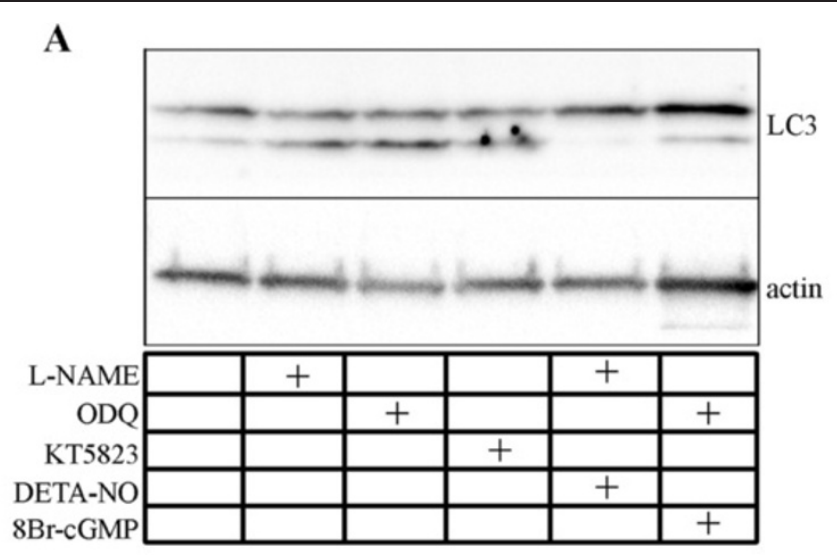

B p62 Bnip3 Atg 4
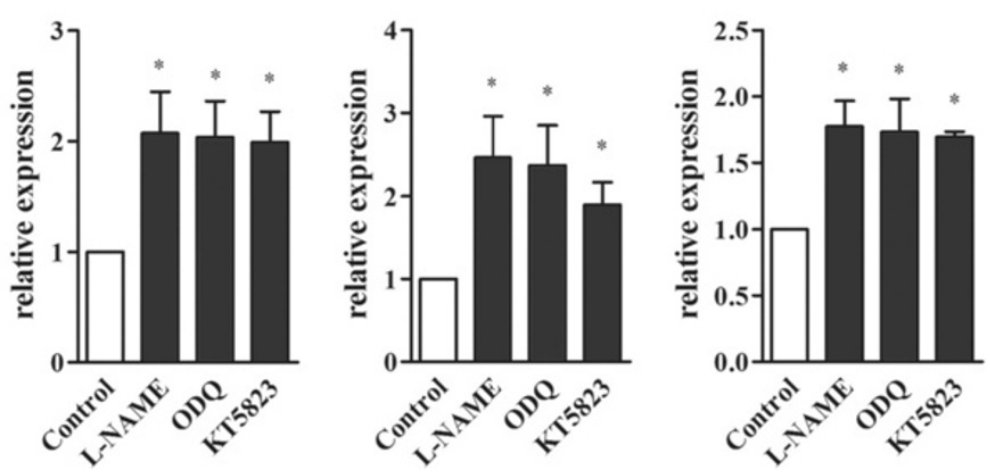

Figure 4 NO signalling and autophagic pathway on myogenic precursor cells. (A) Western blot analysis of LC3 lipidation in cells differentiated for six hours in the absence or in the presence of L-NAME $(6 \mathrm{mM})$, ODQ $(10 \mu \mathrm{M}), \mathrm{KT} 5823(1 \mu \mathrm{M})$, L-NAME + DETA-NO (80 $\mu \mathrm{M})$, and $\mathrm{ODQ}+8 \mathrm{Br}-\mathrm{CGMP}(2.5 \mathrm{mM})$. Actin was used as the internal standard. Image is representative of at least five independent experiments. (B) $\mathrm{qPCR}$ analysis of mRNA levels for p62, Bnip3 and Atg4 in cells differentiated for six hours in the absence (control) or in the presence of L-NAME ODQ, and KT5823. Values are expressed as the fold change over control. Each histogram represents the data obtained from at least five independent experiments. ${ }^{*} P<0.05$ versus respective control.

Additional file 2: Figure S2C, the relative mass of the muscles for the P120 NOS1-/- mice was significantly lower than the relative mass of the muscles for the control mice. This excludes the possibility that the changes in muscle mass are simply due to an overall change in size of the mice.

The overall morphology of the tibialis anterior and diaphragm muscle in P120 NOS1-/- mice was normal, without pathological features of necrosis, macrophage infiltration and centronucleated fibres (see Additional file 2: Figure S2D). In addition, the number of fibres in tibialis anterior muscles was comparable in both NOS1-/- and control mice (Figure 6B). By contrast, laminin staining of tibialis anterior and diaphragm, used to identify individual muscle fibres, revealed a significant decrease in the mean CSA of tibialis anterior and diaphragm sections in P120 NOS1-/- mice when compared with control (Figure 6C-H).
The examination of multiple time points was then carried out in order to establish a possible link between the changes in mitochondrial homeostasis and the reduction in muscle size. The CSA (Figure 7A-C) and the number of myonuclei (Figure 7D) of hind limb muscle fibres were significantly decreased in P10 NOS1-/mice, when compared with the respective control. Muscle growth during post-natal development (P0 to P21), but not at later stages, is accompanied by a continuous increase in the number of myonuclei resulting from satellite cell fusion $[69,73]$. As shown in Figure 7E, NOS1-/ - cells exhibited lower levels of myosin and MyoD, which are markers of myogenic differentiation, as compared to control cells. Interestingly, CycloD staining of differentiating myogenic precursor cells indicated that the absence of $n N O S \mu$ induces diffuse mitochondrial fragmentation (Figure 7F) [25]. Taken together, our data argue that the absence of $n N O S \mu$ induces mitochondrial 


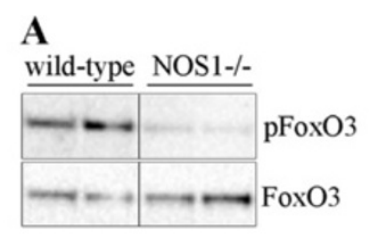

C

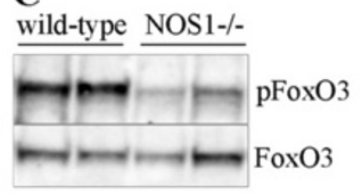

E

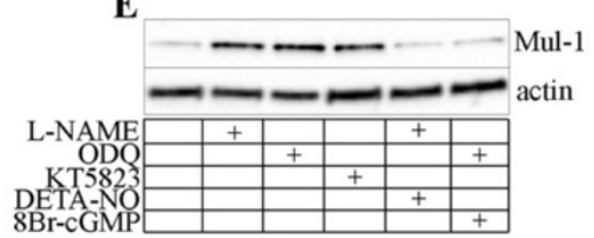

B

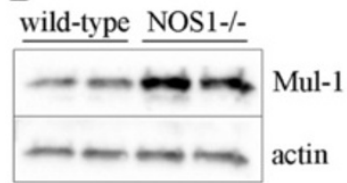

D

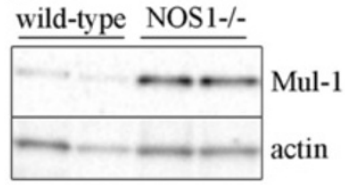

F

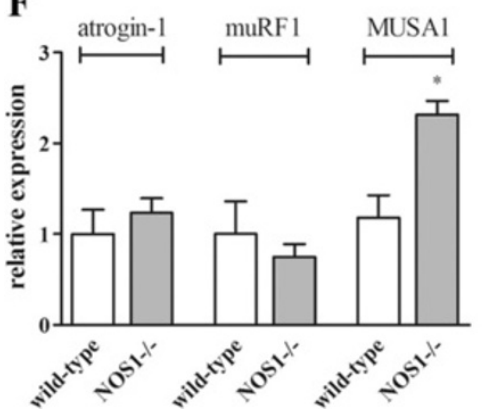

G

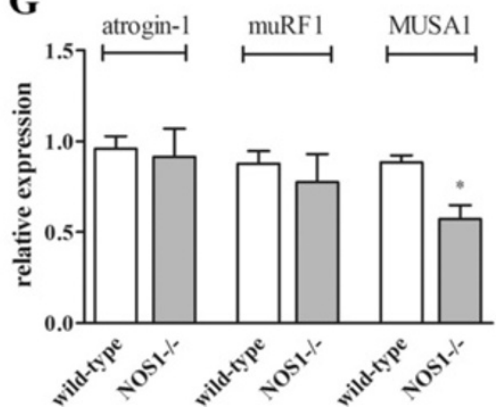

Figure 5 NO signalling, FoxO3, and ubiquitin ligases. Western blot analysis of phosphorylated FoxO3 levels (pFoxO3) or mitochondrial ubiquitin ligase Mul-1 expression in tibialis anterior (A-B) and diaphragm (C-D) of wild-type and NOS1-/- mice at P120. FoxO3 or actin were used as the internal standard. The images are representative of results obtained from at least four to ten different animals per experimental group. (E) Western blot analysis of Mul-1 expression in myogenic precursor cells differentiated in the absence or in the presence of L-NAME (6 mM), ODQ $(10 \mu M), K T 5823(1 \mu M)$, L-NAME + DETA-NO $(80 \mu \mathrm{M})$ and ODQ +8 Br-cGMP $(2.5 \mathrm{mM})$. Actin was used as the internal standard. The image is representative of at least five independent experiments. QPCR analysis of mRNA levels for atrogin-1, muRF1 and MUSA1 in tibialis anterior (F) and diaphragm (G) muscles of wild-type and NOS1-/- mice at P120. Values are expressed as the fold change over wild-type. Each histogram represents the data obtained from at least five to eight different animals per experimental group. ${ }^{*} P<0.05$ versus the respective wild-type control.

fragmentation and a deficit in satellite cell fusion/differentiation, thus impairing fibre growth.

At P30 we found that the CSA of tibialis anterior was significantly decreased in NOS1-/- mice, when compared with controls (Figure 8A-C). In this crucial time of muscle growth we also measured the activation of the Akt-mTOR pathway as a positive regulator $[55,69,73,74]$. As shown in Figure 8D, phosphorylated levels of S6 ribosomal protein, 4E-BP1 and Akt in tibialis anterior muscles of NOS1-/- mice were lower than in the controls. FoxO3 proteins are phosphorylated by Akt, which renders them inactive; this may explain why phosphorylated FoxO3 levels were found to be lower as well, while Mul1 was overexpressed (Figure 8E). Of importance, both events are correlated with muscle mitochondrial dysfunction and growth $[29,55,68,69,73,74]$.
Using NOS1-/- mice it has been previously shown that nNOS modulates the mechanism of disuse-induced atrophy via FoxO transcription factors [75]. Our observation that at P10, P30 (see Additional file 2: Figure S2E-F) and P120 (Figure 5E-F) NOS1-/- and control mice expressed similar levels of transcripts encoding the classical atrogenes atrogin- 1 and MuRF1 $[69,70,75]$, indicates that the atrophy pathways do not play a key role in the development of NOS1-/- muscles.

\section{nNOS $\mu$ deficiency affects muscle function}

We evaluated whether the absence of nNOS $\mu$ affected skeletal muscle function. The WBT measurement determines the total phasic forward pulling tension exerted by the fore and hind limb muscles and reflects the maximal acute phasic force the mouse can achieve to escape 


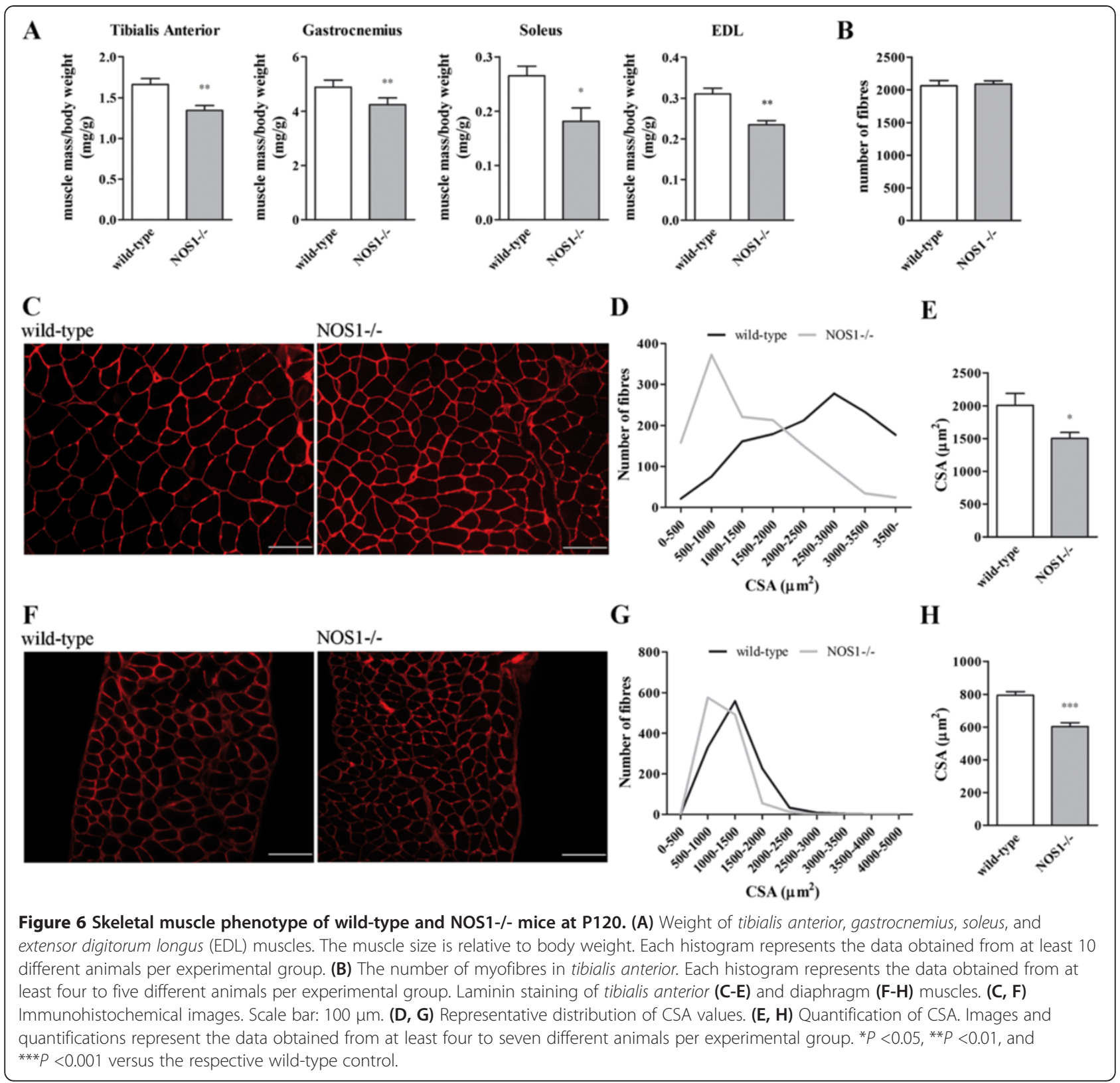

a potentially harmful event [52]. As shown in Figure 9A, the WBT normalised for body weight in P120 NOS1-/mice was significantly lower than in the wild-type control, consistent with an unpaired muscle specific force output in the absence of $\mathrm{nNOS} \mu$.

We also examined the muscle resistance to fatigue: we subjected NOS1-/- mice to treadmill running, that measures resistance to fatigue during a forced exercise, and examined both exercise performance and tolerance. As shown in Figure 9B, the total distance run by NOS1-/mice during one bout of exhaustive treadmill running (day $1)$ was significantly lower when compared to controls. This reduction in performance of NOS1-/- mice was also observed after repeated challenges: NOS1-/- mice showed significant exercise intolerance after repetitive exercise challenges, while control mice at day 3 showed even improved exercise capacity, compared to day 1. NOS1-/mice also exhibited a significantly decreased treadmill runtime to exhaustion (Figure 9C).

We then assessed the structure/damage of skeletal muscle myofibres after exercise. TEM analysis performed in tibialis anterior muscles of P120 NOS1-/- mice after the treadmill running showed marked ultrastructural changes, as, for instance, defects in the organisation of the contractile apparatus (sarcomere), that were observed neither in the wild-type mice nor in unchallenged NOS1-/- mice (Figure 9D). The features observed in challenged NOS1-/mice might be a direct consequence of denervation events 


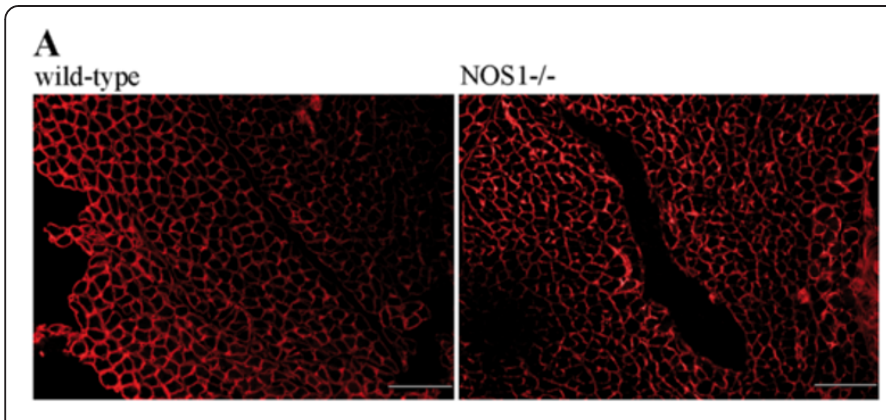

D

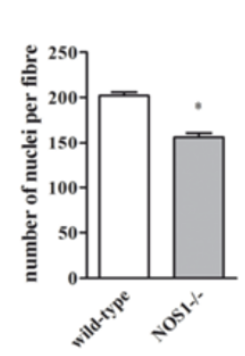

E

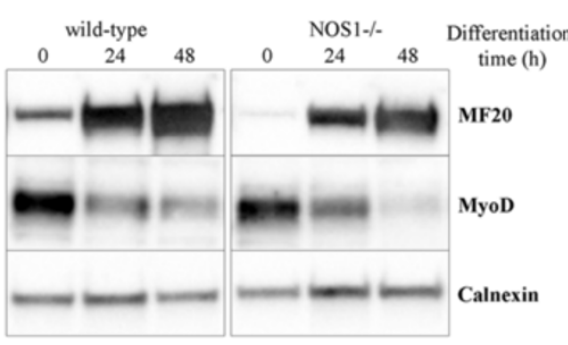

B

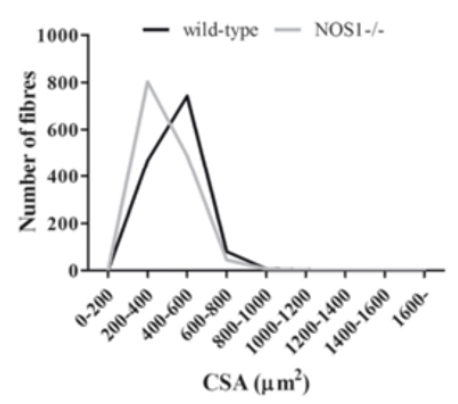

F wild-type

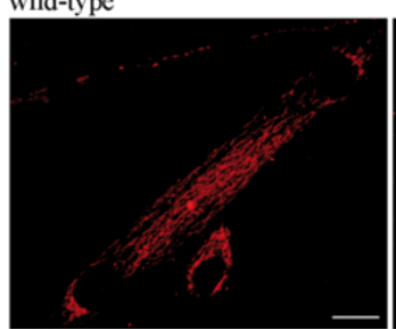

C

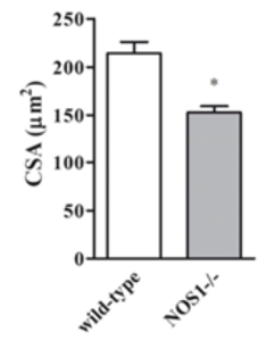

Figure 7 Skeletal muscle phenotype of wild-type and NOS1-/- mice at P10. (A-C) Laminin staining of hind limb muscles. (A) Immunohistochemical images. Scale bar: $100 \mu \mathrm{m}$. (B) Representative distribution of CSA values. (C) Quantification of CSA. Images and quantifications represent the data obtained from at least five different animals per experimental group. (D) Number of myonuclei per fibre in hind limb muscles. Each histogram represents the data obtained from at least three different animals per experimental group. (E) Western blot analysis of myosin (MF20) and MyoD expression in myogenic precursor cells isolated from wild-type and NOS1-/- mice and differentiated for increasing times. Calnexin was used as the internal standard. Images are representative of at least three independent experiments. (F) Confocal microscopy imaging of myogenic precursor cells isolated from wild-type and NOS1-/- mice and differentiated for 48 hours. Mitochondrial morphology was detected by mitochondrial matrix-specific protein cyclophillin D staining. Scale Bar: $10 \mu \mathrm{m}$. Images are representative of at least three independent experiments. ${ }^{*} P<0.05$ versus the respective wild-type control.

as also indicated by collagen fibres deposition and motor end-plates lacking the presynaptic nerve ending (data not shown). As shown in Figure 9E, tibialis anterior muscles of P120 NOS1-/- mice after the treadmill running displayed an increased uptake versus wild-type of EBD, which stains damaged myofibres [47]. As an in vivo indicator of skeletal muscle damage we also analysed the serum levels of CK, a skeletal muscle enzyme released during fibre degeneration whose activity increased in dystrophic animals $[16,18]$. As expected, in NOS1-/- mice after the treadmill running, the serum CK activity was found to be significantly higher than that in the wild-type mice (Figure 9F).

\section{Discussion}

This study documents that $\mathrm{nNOS} \mu$ deficiency, while severely altering the structure and bioenergetics potential of skeletal muscle mitochondria does not impact significantly on the overall resting muscle structure, apart from reducing muscle mass and the CSA of the myofibres of specific muscles. When the muscle is exposed to workloads, however, the consequences of $\mathrm{nNOS} \mu$ deficiency become apparent, with a significantly reduced resistance of the muscles accompanied by increased sensitivity to exercise-induced damage. This establishes for the first time a link between a deficit in NO signalling, mitochondrial alterations and skeletal muscle impairments.

The first result emerging from our analysis is that nNOS $\mu$ deficiency is per se sufficient to induce profound defects in mitochondria, with alterations in mitochondrial distribution, shape, morphology and size accompanied by a latent mitochondrial dysfunction such that energy generation is impaired. Nitric oxide has several key functions in mitochondria: it inhibits mitochondrial fission, induces mitochondrial biogenesis and controls mitochondrial respiratory rate by reversible inhibition of complex IV in the mitochondrial respiratory chain $[25,76,77]$. Furthermore, it controls the expression of several enzymes in the Krebs cycle [78]. Derangement of these mitochondrial functions is most likely at the basis of the multiple mitochondrial deficits we observed in NOS1-/- mice.

Of importance, we found that this overall mitochondrial dysfunction was accompanied both in intact myofibres in vivo and in isolated satellite cells in vitro by an enhanced $U P R^{\mathrm{mt}}$ response. It has been hypothesised that the $U P R^{\mathrm{mt}}$ is activated prior to the induction of autophagy [79]; in particular, that the autophagy pathway is activated when mitochondria cannot maintain a polarised membrane potential despite $\mathrm{UPR}^{\mathrm{mt}}$ activation. We found 


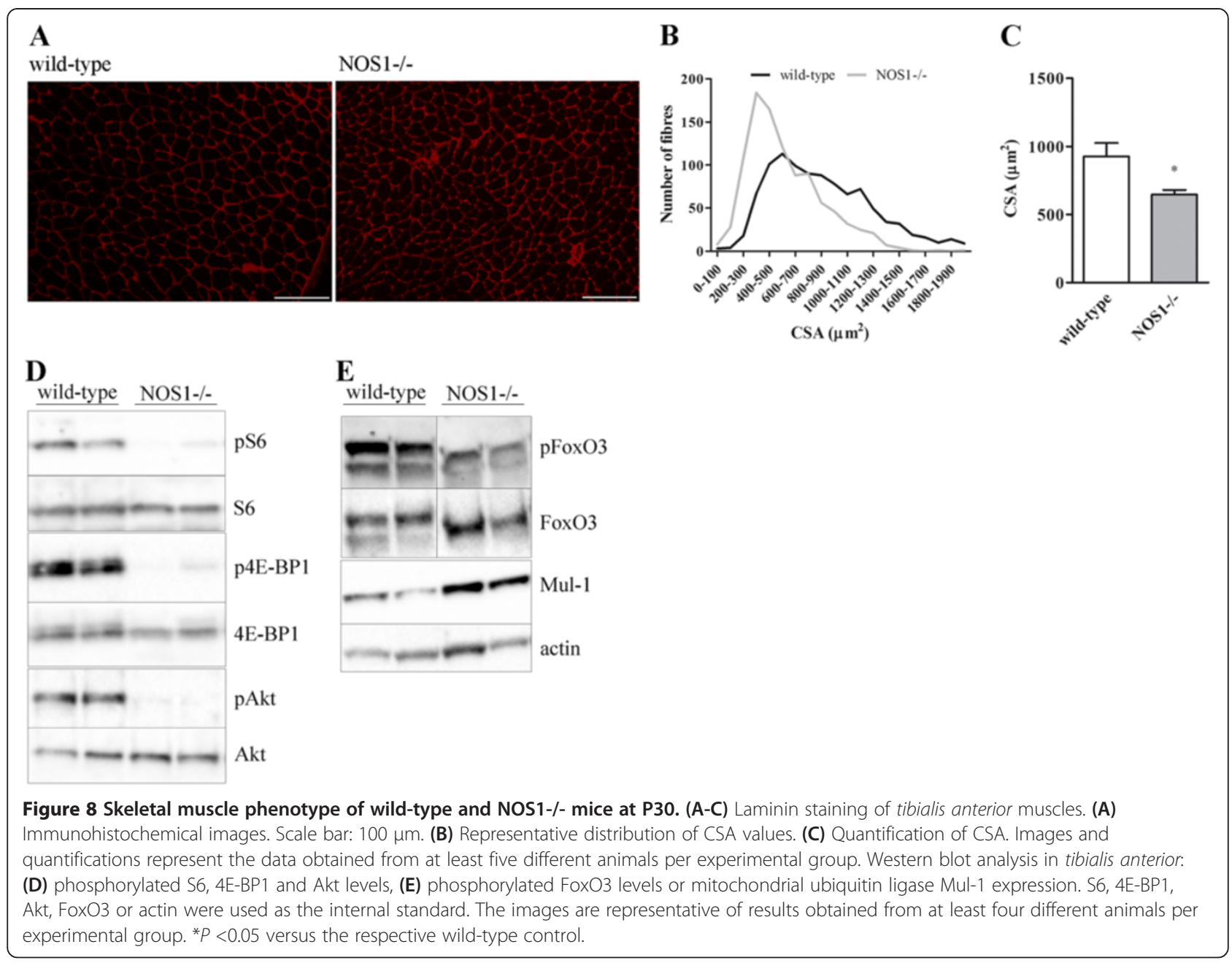

that the increase in $\mathrm{UPR}^{\mathrm{mt}}$ was accompanied by autophagy and increased expression of molecules relevant to autophagic signalling, namely p62, Bnip3 and Atg4. This suggests that $\mathrm{nNOS} \mu$ deficiency leads to a sufficiently severe mitochondrial deficit that cannot be restored by $U P R^{\mathrm{mt}}$. The enhanced autophagic and $\mathrm{UPR}^{\mathrm{mt}}$ response were normalised when the CGMP-dependent signalling was activated, indicating that these events are controlled by NO via its physiological second messenger cGMP.

The second relevant information is that an altered NO system leads to impairment of muscle function that is selective to specific parameters and unmasked during exercise. In particular we found that skeletal muscles in the absence of $n N O S \mu$ are smaller relative to the rest of the body, thus indicating that muscle mass decrease was not simply attributable to a generalised decreased body mass tissues (including adipose tissue) and likely due to a specific reduction in the size of the muscle fibres themselves. In agreement with this, NOS1-/- mice muscles (that is, tibialis anterior and diaphragm) displayed smaller myofibre CSA when compared to littermate controls, although they did not show any pathological features reminiscent of muscle damage, such as inflammation, necrosis or fibrosis. Similar morphological data were obtained in male NOS1-/- mice backcrossed onto the B6129 background (our experimental model) [71] or backcrossed onto the C57BL/6 background [80], although in the latter model no difference in tibialis anterior muscle mass relative to body mass was reported. That the decrease in muscle mass is due to mechanisms other than the decrease in body mass was recently suggested using NOS1-/- $m d x$ mice [72]. The deficiency of $\mathrm{nNOS} \mu$ is also accompanied by muscle ageing [81] and fibre growth was prevented in the NOS1-/- mice model of skeletal muscle hypertrophy [82] and NOS1-/- $m d x$ mice [72]. In a recent study, no difference in the weight and CSA of tibialis anterior muscles from NOS1-/- and control was also reported but the animal background was not indicated [83]. Discrepancies in these studies may be explained, at least in part, by strain-specific modulation of the nNOS $\mu$-regulated phenotype, a hypothesis substantiated by the observation, by the same 
A

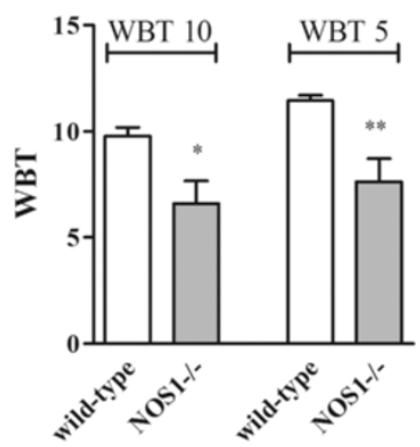

B

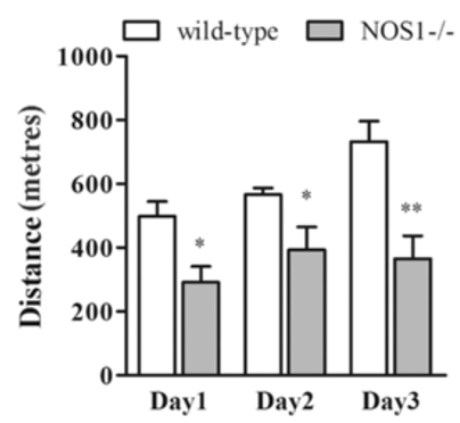

C

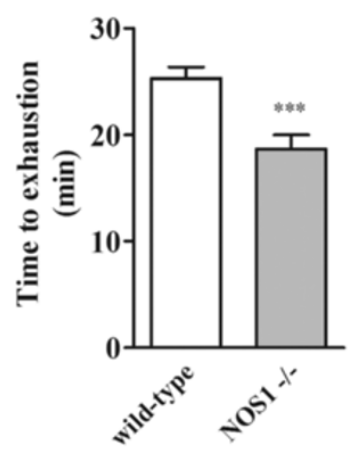

D

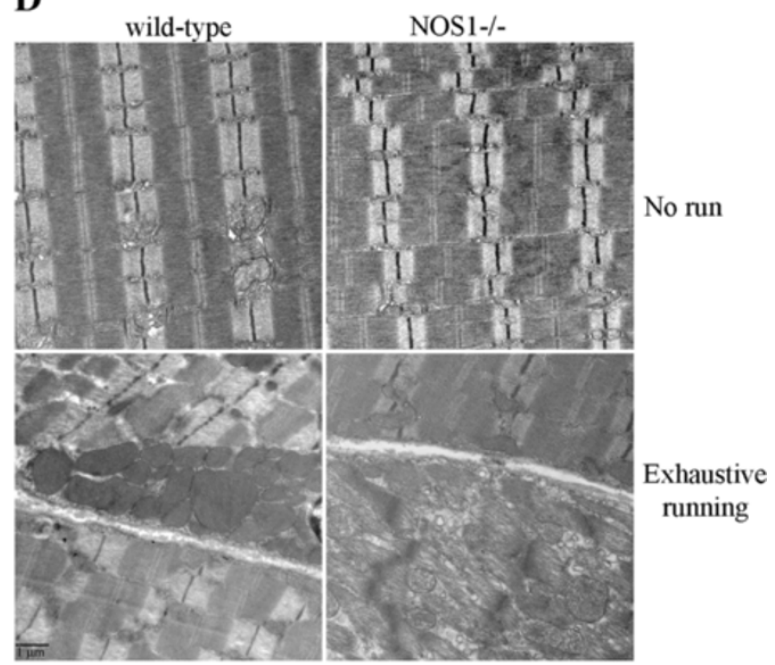

F
E

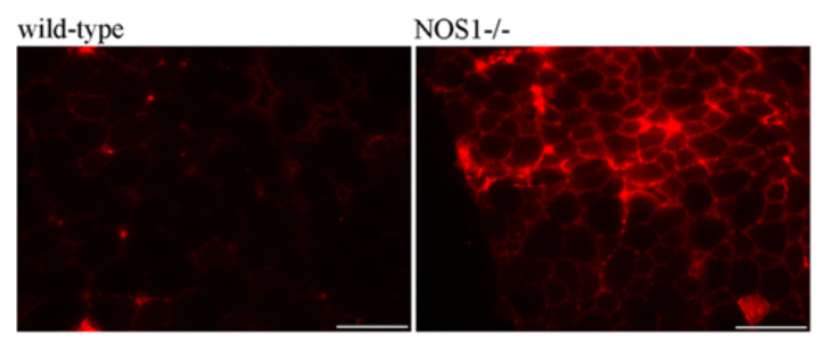

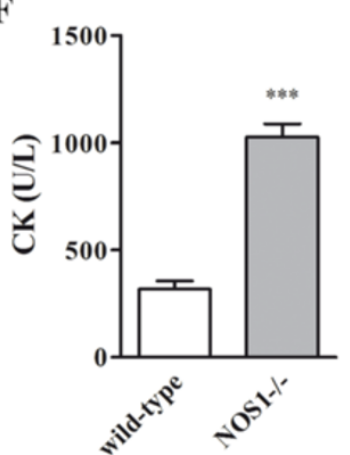

Figure 9 Skeletal muscle function in wild-type and NOS1-/- mice. (A) WBT measurements determined by dividing the average of the top ten or top five forward pulling tensions, respectively, by the body weight. (B) Running distance calculated during one bout of exhaustive treadmill running (day 1) and after repeated challenges (days 2 and 3). (C) Treadmill runtime to exhaustion calculated as the averages obtained at day 1 to 3. Each histogram represents the data obtained from at least four to five different animals per experimental group. (D) TEM analysis performed in tibialis anterior muscles of both unchallenged (no run) and challenged (exhaustive running) mice. The images are representative of results obtained from at least three different animals per experimental group. (E) EBD uptake in tibialis anterior muscles after the treadmill running. Scale Bar: $100 \mu \mathrm{m}$. The images are representative of results obtained from at least four different animals per experimental group. (F) CK serum levels (units per litre) of mice after treadmill running. Each histogram represents the data obtained from at least four different animals per experimental group. ${ }^{*} P<0.05$, ${ }^{* *} P<0.01$ and ${ }^{* * *} P<0.001$ versus the respective wild-type control. WBT and treadmill running were performed on animals at P120.

group, that morphological data differed between NOS1-/mice backcrossed onto the C57BL/6 and the B6129 background $[37,71,80]$.
The functional studies revealed two important aspects of the role of $\mathrm{NO}$ in skeletal muscle. Firstly, the fact that NOS1-/- mice in our in vivo experiments exhibited a 
deficit in forward pulling tension and resistance to fatigue during a forced exercise indicates that $n N O S \mu$ is important to maintain skeletal muscle strength and the animal's ability to perform in repetitive exercise training. Our results in vivo are in line with a previous study with an in situ approach reporting that $\mathrm{nNOS} \mu$-deficient tibialis anterior muscles exhibit a reduced force production and a specific deficit in adapting to exercise and develop profound fatigue upon repeated contraction [71]. An excessive fatigue has been also observed in NOS1-/- mice and wild-type mice treated with a nNOS inhibitor [12]. A specific and intrinsic deficit in muscle force production has been recently reported in NOS1-/- $m d x$ mice, although muscle fatigue was unaffected by nNOS depletion [72]. Secondly, our data on muscle phenotype and CK measurements after treadmill running indicate that nNOS $\mu$ deficiency induces muscle degeneration/damage post-exercise. This raises the possibility that $\mathrm{nNOS} \mu-$ deprived muscles cannot activate protective responses. Accordingly, NOS1-/- $m d x$ mice displayed increased susceptibility to eccentric contraction-induced muscle damage [72]. In addition, expression of a muscle-specific nNOS transgene prevents muscle membrane injury during modified muscle use [84]. In this respect, there is a general agreement that $\mathrm{NO}$ produced by nNOS plays an important role in muscle repair in chronic conditions $[5,8,9]$ although the use of NOS1-/- mice suggested that nNOS is not essential to functional recovery after acute injury [80].

The third important observation is the correlation between mitochondrial defects and muscle impairment. Alterations in the content, shape or function of the mitochondria appear to occur in damaged muscle and inhibition of mitochondrial fission protects from muscle loss during fasting [29]. Recent findings have also underlined the crucial role of autophagy in the control of muscle mass and functions $[29,31,55,69]$. Autophagy derangement is involved in a number of inherited muscle diseases [31-33]. Of interest, mitochondria are involved in regulating autophagy [30]. In addition, skeletal muscle was shown to be sensitive to the physiological stressors that trigger the $\mathrm{UPR}^{\mathrm{mt}}[35,36]$ and $\mathrm{UPR}^{\mathrm{mt}}$ is activated in skeletal muscle during exercise as part of an adaptive response to exercise training [54]. Here, we raise the possibility that mitochondrial dysfunction, $\mathrm{UPR}^{\mathrm{mt}}$ and autophagy are functionally related to each other and promoted by a single event, that is, the deficit in NO signalling, thus suggesting that the association of altered mitochondrial homeostasis and muscle phenotype/performance in NOS1-/- mice is not coincidental.

The experiments we carried-out in myogenic precursor cells and NOS1-/- mice during critical stages of muscle development are consistent with an association of altered mitochondrial homeostasis and muscle phenotype/ performance and provide an indication of the mechanism responsible for the impaired fibre growth resulting in a deficit of muscle performance. In particular, nNOS $\mu$ absence altered mitochondrial homeostasis in myogenic precursor cells with a decrease in the number of myonuclei per fibres and impaired muscle development at early stages of growth. This also suggests that fusion of myogenic precursor cells during perinatal myogenesis is impaired. Accordingly, NO has been shown to stimulate the ability of myogenic precursor cells to become activated and fuse to each other $[5,8,85]$. There is a general agreement that mitochondria change when the myoblasts differentiate into myotubes [27]. Also, NO maintains functional mitochondria and this permits differentiation of myogenic precursor cells in vitro [25]. At the signalling level, the Akt-mTOR pathway and Akt-FoxO3-Mul-1 axis are involved in skeletal muscle growth/wasting, autophagy and mitochondrial dysfunction $[29,31,38,46,55,58,67-69,73,74]$. Of interest, Mul-1 has been recently reported to be upregulated during muscle wasting, possibly via an autophagic mechanism involving FoxO3 transcription factors [68]. Our data indicate the relevance of the above signalling pathways and that they are controlled by NO. We observed an inhibition of the AktmTOR pathway in the absence of $\mathrm{nNOS} \mu$. Concomitantly, the Akt-FoxO3-Mul-1 axis was also dysregulated. In addition, the inhibition of the nNOS/NO/cGMP/PKG system induced the transcriptional activity of FoxO3 and increased Mul-1 expression. These events are likely associated with nNOS $\mu$-dependent impairments of muscle fibre growth.

We cannot exclude that failure of other NO-dependent action involving, for instance, the vascular system, may have contributed to the functional and structural defects we observed in skeletal muscle. Extensor digitorum longus of NOS1-/- mice revealed an altered capillary-to-fibre ratio but not changes in the capillary ultrastructure or the hemodynamics at basal conditions [86]. Noteworthy, NO generated by sarcolemmal $\mathrm{nNOS} \mu$ normally acts as a paracrine signal that optimises blood flow in the working muscle $[12,87,88]$ and the protective vasodilating action is impaired in the contracting muscles of NOS1-/- mice $[12,89]$. In this respect, the lack of this vasodilating action in NOS1-/- mice has been suggested to affect muscle performance [71]. Results obtained in NOS1-/- mice with different cardiac injuries indicated a protective role of nNOS, although an opposite effect cannot be excluded [90,91]. The deficit in exercise performance of NOS1-/- muscles may be the consequence, at least in part, of a decreased oxygen delivery following blood flow impairment.

\section{Conclusions}

Muscle exercise performance is a complex physiological process that can occur by many different mechanisms and NO has long been described to be relevant among 
them [2]. Our study now suggests that the relevance of $\mathrm{NO}$ also resides in the fact that it regulates key homeostatic mechanisms in skeletal muscle, namely mitochondrial bioenergetics and network remodelling, $\mathrm{UPR}^{\mathrm{mt}}$ and autophagy. Although NOS1-/- mice do not display the overt features of myopathies, such as muscle degeneration, reactive regeneration and replacement of muscle with fibroadipous tissue $[92,93]$, we clearly show that alterations of the NO system significantly impair muscle fibre growth, thus resulting in a deficit of muscle force and the ability to sustain prolonged exercise. This aspect may explain why NO deficiency contributes to muscle impairment in degenerative disease of the muscle, such as muscular dystrophies.

\section{Additional files}

Additional file 1: Figure S1. Mitochondrial ultrastructure and LC3 lipidation in skeletal muscles of wild-type and NOS1-/- mice. (A) TEM i mages of subsarcolemmal mitochondria of tibialis anterior muscles. Scale bar: $0.1 \mu \mathrm{m}$. (B) TEM images of intermyofibrillar mitochondria of tibialis anterior muscles. Scale bar: $1 \mu \mathrm{m}$. (C) TEM images of diaphragm muscles detecting the presence of autophagic vacuoles (arrowheads) in NOS1-/ fibres. TEM images are representative of results obtained from at least three different animals per experimental group. (D) Western blot analysis of LC3 lipidation in diaphragm muscles of wild-type and NOS1-/- mice. GAPDH was used as internal standard. The image is representative of results obtained from at least 10 different animals per experimental group. Analyses were performed on animals at P120.

Additional file 2: Figure S2. Weight, muscle structure and muscle expression of ubiquitin ligases in wild-type and NOS1-/- mice. Body (A) and visceral adipose tissue (VAT) (B) weight. Each histogram represents the data obtained from at least three to eight different animals per experimental group. ${ }^{*} P<0.05$, and ${ }^{* *} P<0.01$ versus the respective wild-type control. (C) Pictures of tibialis anterior, soleus, and gastrocnemius muscles. The image is representative of at least 10 different animals per experimental group. (D) Histological sections of tibialis anterior and diaphragm muscles stained with $\mathrm{H} \& \mathrm{E}$. The images are representative of results obtained from at least five different animals per experimental group. Scale bar: $100 \mu \mathrm{m}$. Analyses were performed on animals at P120. qPCR analysis of mRNA levels for atrogin-1 and muRF1 in hind limb muscles at P10 (E) and tibialis anterior muscles at P30 (F). Values are expressed as the fold change over wild-type. Each histogram represents the data obtained from at least five different animals per experimental group.

\section{Abbreviations}

CGMP: cyclic guanosine monophosphate; CK: creatine kinase; CSA: cross sectional area; EBD: Evans blue dye; EDTA: ethylenediaminetetraacetic acid; EGTA: ethyleneglycoltetraacetic acid; FCCP: protonophore carbonylcyanidep-trifluoromethoxyphenyl hydrazone; H \& E: haematoxylin and eosin; L-NAME: $L^{\omega}$-arginine methylester; mTOR: mammalian target of rapamycin; mtDNA: mitochondrial DNA; Mul-1: mitochondrial E3 ubiquitin protein ligase 1; nNOS: NO synthases; NO: nitric oxide; OXPHOS: oxidative phosphorylation; P10/30/120: postnatal day 10/30/120; PKG: cGMP-dependent-protein kinases; qPCR: real-time quantitative PCR; SDS: sodium dodecyl sulphate TEM: transmission electron microscopy; TMRM: tetramethylrhodamine, methyl ester; UPR ${ }^{\mathrm{mt}}$ : unfolded protein response; WBT: whole body tension; YFP: yellow fluorescent protein

\section{Competing interests}

The authors declare that they have no competing interests.

\section{Authors' contributions}

CDP was responsible for conception and design of the study, acquisition of data, analysis and interpretation of data, and revising the manuscript. FM, SP,
$E A, T T, S R, V R, S C, V C$ and PP acquired and analysed the data. CM, MTB and MS analysed the data and revised the manuscript. CP participated in the design of the study, analysed and interpreted the data, and revised the manuscript. DC and EC participated in the design and the coordination of the study, analysed and interpreted the data, drafted and revised the manuscript, and wrote the final version of the manuscript. All authors read and approved the final manuscript.

\section{Authors' information}

CDP is a post-doctoral research associate. FM and SP are PhD students. SR is a research fellow. EA, TT, VR, SC and VC are post-doctoral research fellows. PP is a graduate medical student. MTB is a Senior Researcher. CM is a Professor of Human Anatomy. MS is a Professor of Pathology. CP is a Professor of Pharmacology. DC is a Professor of Physiology. EC is a Professor of Pharmacology and the Head of the Pharmacology group.

\section{Acknowledgements}

We thank Laura Pozzi (Scientific Institute IRCCS Eugenio Medea, Bosisio Parini, Lecco, Italy) for technical help. We are grateful to Prof. Luca Scorrano (University of Padova, Padova, Italy) for providing us with pDsRed2-Mito. This work was supported by: "Ministero della Salute" "Giovani Ricercatori 2011-2012" grant to C. D.P and "Ricerca corrente 2014" grant to E.C.; "Ministero dell'Istruzione, Università e Ricerca", PRIN2010-2011 grants to E.C. and D.C.; European Community's framework programme FP7/2007-2013 under the agreement n²23098 (OPTISTEM) and $n^{\circ} 241440$ (ENDOSTEM) to E.C. The funders had no role in study design, data collection and analysis, decision to publish, or preparation of the manuscript.

\section{Author details}

'Unit of Clinical Pharmacology, National Research Council-Institute of Neuroscience, Department of Biomedical and Clinical Sciences "Luigi Sacco", University Hospital "Luigi Sacco", Università di Milano, Milano, Italy. ${ }^{2}$ Scientific Institute IRCCS Eugenio Medea, Bosisio Parini, Italy. ${ }^{3}$ Dulbecco Telethon Institute at Venetian Institute of Molecular Medicine, Padova, Italy. ${ }^{4}$ National Research Council-Institute of Neuroscience, Department of Medical Biotechnology and Translational Medicine, Università di Milano, Milano, Italy. ${ }^{5} \mathrm{CNI@NEST}$, Italian Institute of Technology, Pisa, Italy. ${ }^{6}$ Unit of Morphology, Department of Biomedical and Clinical Sciences "Luigi Sacco", Università di Milano, Milano, Italy. ${ }^{7}$ Department of Biomedical Science, Università di Padova, Padova, Italy. ${ }^{8}$ Department for Innovation in Biological, Agro-food and Forest Systems, Università della Tuscia, Viterbo, Italy.

Received: 26 June 2014 Accepted: 18 November 2014

Published online: 12 December 2014

\section{References}

1. Alderton WK, Cooper CE, Knowles RG: Nitric oxide synthases: structure, function and inhibition. Biochem J 2001, 357:593-615.

2. Stamler JS, Meissner G: Physiology of nitric oxide in skeletal muscle. Physiol Rev 2001, 81:209-237.

3. Brenman JE, Chao DS, Xia H, Aldape K, Bredt DS: Nitric oxide synthase complexed with dystrophin and absent from skeletal muscle sarcolemma in Duchenne muscular dystrophy. Cell 1995, 82:743-752.

4. Rubinstein I, Abassi Z, Coleman R, Milman F, Winaver J, Better OS: Involvement of nitric oxide system in experimental muscle crush injury. J Clin Invest 1998, 101:1325-1333.

5. De Palma C, Clementi E: Nitric oxide in myogenesis and therapeutic muscle repair. Mol Neurobiol 2012, 46:682-692.

6. McConell GK, Rattigan S, Lee-Young RS, Wadley GD, Merry TL: Skeletal muscle nitric oxide signaling and exercise: a focus on glucose metabolism. Am J Physiol Endocrinol Metab 2012, 303:E301-307.

7. Eu JP, Hare JM, Hess DT, Skaf M, Sun J, Cardenas-Navina I, Sun QA, Dewhirst M, Meissner G, Stamler JS: Concerted regulation of skeletal muscle contractility by oxygen tension and endogenous nitric oxide. Proc Natl Acad Sci U S A 2003, 100:15229-15234.

8. Buono R, Vantaggiato C, Pisa V, Azzoni E, Bassi MT, Brunelli S, Sciorati C, Clementi E: Nitric oxide sustains long-term skeletal muscle regeneration by regulating fate of satellite cells via signaling pathways requiring Vangl2 and cyclic GMP. Stem Cells 2012, 30:197-209. 
9. Cordani N, Pisa V, Pozzi L, Sciorati C, Clementi E: Nitric oxide controls fat deposition in dystrophic skeletal muscle by regulating fibro-adipogenic precursor differentiation. Stem Cells 2014, 32:874-885.

10. Chao DS, Gorospe JR, Brenman JE, Rafael JA, Peters MF, Froehner SC, Hoffman EP, Chamberlain JS, Bredt DS: Selective loss of sarcolemmal nitric oxide synthase in Becker muscular dystrophy. J Exp Med 1996, 184:609-618.

11. Crosbie RH, Barresi R, Campbell KP: Loss of sarcolemma nNOS in sarcoglycan-deficient muscle. FASEB J 2002, 16:1786-1791.

12. Kobayashi YM, Rader EP, Crawford RW, lyengar NK, Thedens DR, Faulkner JA, Parikh SV, Weiss RM, Chamberlain JS, Moore SA, Campbell KP: Sarcolemmalocalized nNOS is required to maintain activity after mild exercise. Nature 2008, 456:511-515.

13. Gucuyener K, Ergenekon E, Erbas D, Pinarli G, Serdaroglu A: The serum nitric oxide levels in patients with Duchenne muscular dystrophy. Brain Dev 2000, 22:181-183

14. Wehling M, Spencer MJ, Tidball JG: A nitric oxide synthase transgene ameliorates muscular dystrophy in mdx mice. J Cell Biol 2001, 155:123-131.

15. Wehling-Henricks M, Oltmann M, Rinaldi C, Myung KH, Tidball JG: Loss of positive allosteric interactions between neuronal nitric oxide synthase and phosphofructokinase contributes to defects in glycolysis and increased fatigability in muscular dystrophy. Hum Mol Genet 2009, 18:3439-3451.

16. Brunelli S, Sciorati C, D'Antona G, Innocenzi A, Covarello D, Galvez BG, Perrotta C, Monopoli A, Sanvito F, Bottinelli R, Ongini E, Cossu G, Clementi E: Nitric oxide release combined with nonsteroidal antiinflammatory activity prevents muscular dystrophy pathology and enhances stem cell therapy. Proc Natl Acad Sci U S A 2007, 104:264-269.

17. Deponti D, Francois S, Baesso S, Sciorati C, Innocenzi A, Broccoli V Muscatelli F, Meneveri R, Clementi E, Cossu G, Brunelli S: Necdin mediates skeletal muscle regeneration by promoting myoblast survival and differentiation. J Cell Biol 2007, 179:305-319.

18. Sciorati C, Buono R, Azzoni E, Casati S, Ciuffreda P, D'Angelo G, Cattaneo D, Brunelli S, Clementi E: Co-administration of ibuprofen and nitric oxide is an effective experimental therapy for muscular dystrophy, with immediate applicability to humans. Br J Pharmacol 2010, 160:1550-1560.

19. D'Angelo MG, Gandossini S, Martinelli Boneschi F, Sciorati C, Bonato S, Brighina E, Comi GP, Turconi AC, Magri F, Stefanoni G, Brunelli S, Bresolin N, Cattaneo D, Clementi E: Nitric oxide donor and non steroidal anti inflammatory drugs as a therapy for muscular dystrophies: evidence from a safety study with pilot efficacy measures in adult dystrophic patients. Pharmacol Res 2012, 65:472-479.

20. Cossu MV, Cattaneo D, Fucile S, Pellegrino PM, Baldelli S, Cozzi V, Capetti A Clementi E: Combined isosorbide dinitrate and ibuprofen as a novel therapy for muscular dystrophies: evidence from Phase I studies in healthy volunteers. Drug Des Devel Ther 2014, 8:411-419.

21. Tengan CH, Rodrigues GS, Godinho RO: Nitric oxide in skeletal muscle: role on mitochondrial biogenesis and function. Int J Mo/ Sci 2012, 13:17160-17184.

22. Nisoli E, Falcone S, Tonello C, Cozzi V, Palomba L, Fiorani M, Pisconti A Brunelli S, Cardile A, Francolini M, Cantoni O, Carruba MO, Moncada S, Clementi E: Mitochondrial biogenesis by NO yields functionally active mitochondria in mammals. Proc Natl Acad Sci U S A 2004, 101:16507-16512.

23. Clementi E, Nisoli E: Nitric oxide and mitochondrial biogenesis: a key to long-term regulation of cellular metabolism. Comp Biochem Physiol A Mol Integr Physiol 2005, 142:102-110.

24. Cleeter MW, Cooper JM, Darleyusmar VM, Moncada S, Schapira AH: Reversible inhibition of cytochrome-C-oxidase, the terminal enzyme of the mitochondrial respiratory-chain, by nitric-oxide - implications for neurodegenerative diseases. FEBS Lett 1994, 345:50-54.

25. De Palma C, Falcone S, Pisoni S, Cipolat S, Panzeri C, Pambianco S, Pisconti A, Allevi R, Bassi MT, Cossu G, Pozzan T, Moncada S, Scorrano L, Brunelli S, Clementi E: Nitric oxide inhibition of Drp1-mediated mitochondrial fission is critical for myogenic differentiation. Cell Death Differ 2010, 17:1684-1696.

26. Russell AP, Foletta VC, Snow RJ, Wadley GD: Skeletal muscle mitochondria: A major player in exercise, health and disease. Biochim Biophys Acta 1840, 2014:1276-1284

27. Wagatsuma A, Sakuma K: Mitochondria as a potential regulator of myogenesis. ScientificWorldJournal 2013, 2013:593267.

28. Eisner V, Lenaers G, Hajnoczky G: Mitochondrial fusion is frequent in skeletal muscle and supports excitation-contraction coupling. J Cell Biol 2014, 205:179-195.
29. Romanello V, Guadagnin E, Gomes L, Roder I, Sandri C, Petersen Y, Milan G, Masiero E, Del Piccolo P, Foretz M, Scorrano L, Rudolf R, Sandri M: Mitochondrial fission and remodelling contributes to muscle atrophy. EMBO J 2010, 29:1774-1785.

30. Gomes LC, Di Benedetto G, Scorrano L: During autophagy mitochondria elongate, are spared from degradation and sustain cell viability. Nat Cell Biol 2011, 13:589-598.

31. Sandri M, Coletto L, Grumati P, Bonaldo P: Misregulation of autophagy and protein degradation systems in myopathies and muscular dystrophies. J Cell Sci 2013, 126:5325-5333.

32. Grumati $P$, Coletto L, Sandri M, Bonaldo P: Autophagy induction rescues muscular dystrophy. Autophagy 2011, 7:426-428.

33. Grumati P, Coletto L, Sabatelli P, Cescon M, Angelin A, Bertaggia E, Blaauw B, Urciuolo A, Tiepolo T, Merlini L, Maraldi NM, Bernardi P, Sandri M, Bonaldo P: Autophagy is defective in collagen VI muscular dystrophies, and its reactivation rescues myofiber degeneration. Nat Med 2010, 16:1313-1320

34. Haynes CM, Ron D: The mitochondrial UPR - protecting organelle protein homeostasis. J Cell Sci 2010, 123:3849-3855

35. Acosta-Alvear D, Zhou Y, Blais A, Tsikitis M, Lents NH, Arias C, Lennon CJ, Kluger $Y$, Dynlacht BD: XBP1 controls diverse cell type- and conditionspecific transcriptional regulatory networks. Mol Cell 2007, 27:53-66.

36. Iwawaki T, Akai R, Kohno K, Miura M: A transgenic mouse model for monitoring endoplasmic reticulum stress. Nat Med 2004, 10:98-102.

37. Percival JM, Anderson KN, Huang P, Adams ME, Froehner SC: Golgi and sarcolemmal neuronal NOS differentially regulate contraction-induced fatigue and vasoconstriction in exercising mouse skeletal muscle. J Clin Invest 2010, 120:816-826.

38. Mammucari C, Milan G, Romanello V, Masiero E, Rudolf R, Del Piccolo P, Burden SJ, Di Lisi R, Sandri C, Zhao J, Goldberg AL, Schiaffino S, Sandri M: FoxO3 controls autophagy in skeletal muscle in vivo. Cell Metab 2007, 6:458-471.

39. Cervia D, Garcia-Gil M, Simonetti E, Di Giuseppe G, Guella G, Bagnoli P, Dini F: Molecular mechanisms of euplotin C-induced apoptosis: involvement of mitochondrial dysfunction, oxidative stress and proteases. Apoptosis 2007, 12:1349-1363.

40. Kuznetsov AV, Veksler V, Gellerich FN, Saks V, Margreiter R, Kunz WS: Analysis of mitochondrial function in situ in permeabilized muscle fibers, tissues and cells. Nat Protoc 2008, 3:965-976.

41. Votion DM, Gnaiger E, Lemieux H, Mouithys-Mickalad A, Serteyn D: Physical fitness and mitochondrial respiratory capacity in horse skeletal muscle. PLoS One 2012, 7:e34890.

42. Jacobs RA, Boushel R, Wright-Paradis C, Calbet JA, Robach P, Gnaiger E, Lundby $C$ : Mitochondrial function in human skeletal muscle following high-altitude exposure. Exp Physiol 2013, 98:245-255.

43. Perrotta C, Buldorini M, Assi E, Cazzato D, De Palma C, Clementi E, Cervia D: The thyroid hormone triiodothyronine controls macrophage maturation and functions: protective role during inflammation. Am J Pathol 2014, 184:230-247.

44. Livak KJ, Schmittgen TD: Analysis of relative gene expression data using real-time quantitative PCR and the 2-DDCT Method. Methods 2001, 25:402-408.

45. Mouchiroud L, Houtkooper RH, Moullan N, Katsyuba E, Ryu D, Canto C, Mottis A, Jo YS, Viswanathan M, Schoonjans K, Guarente L, Auwerx J: The $\mathrm{NAD}(+) /$ sirtuin pathway modulates longevity through activation of mitochondrial UPR and FOXO signaling. Cell 2013, 154:430-441.

46. Sandri M, Sandri C, Gilbert A, Skurk C, Calabria E, Picard A, Walsh K Schiaffino S, Lecker SH, Goldberg AL: Foxo transcription factors induce the atrophy-related ubiquitin ligase atrogin-1 and cause skeletal muscle atrophy. Cell 2004, 117:399-412.

47. De Palma C, Morisi F, Cheli S, Pambianco S, Cappello V, Vezzoli M, Rovere-Querini P, Moggio M, Ripolone M, Francolini M, Sandri M, Clementi E: Autophagy as a new therapeutic target in Duchenne muscular dystrophy. Cell Death Dis 2012 3:e418.

48. Francolini M, Brunelli G, Cambianica I, Barlati S, Barbon A, La Via L, Guarneri B, Boroni F, Lanzillotta A, Baiguera C, Ettorre M, Buffelli M, Spano P, Clementi F, Pizzi M: Glutamatergic reinnervation and assembly of glutamatergic synapses in adult rat skeletal muscle occurs at cholinergic endplates. J Neuropathol Exp Neurol 2009, 68:1103-1115.

49. Bizzozero L, Cazzato D, Cervia D, Assi E, Simbari F, Pagni F, De Palma C, Monno A, Verdelli C, Querini PR, Russo V, Clementi E, Perrotta C: Acid sphingomyelinase determines melanoma progression and metastatic 
behaviour via the microphtalmia-associated transcription factor signalling pathway. Cell Death Differ 2014, 21:507-520.

50. Armani C, Catalani E, Balbarini A, Bagnoli P, Cervia D: Expression, pharmacology, and functional role of somatostatin receptor subtypes 1 and 2 in human macrophages. J Leukoc Biol 2007, 81:845-855.

51. Sciorati C, Touvier T, Buono R, Pessina P, Francois S, Perrotta C, Meneveri R, Clementi E, Brunelli S: Necdin is expressed in cachectic skeletal muscle to protect fibers from tumor-induced wasting. J Cell Sci 2009, 122:1119-1125.

52. Carlson CG, Rutter J, Bledsoe C, Singh R, Hoff H, Bruemmer K, Sesti J, Gatti F, Berge J, McCarthy L: A simple protocol for assessing inter-trial and inter-examiner reliability for two noninvasive measures of limb muscle strength. J Neurosci Methods 2010, 186:226-230.

53. George Carlson C, Bruemmer K, Sesti J, Stefanski C, Curtis H, Ucran J, Lachey J, Seehra JS: Soluble activin receptor type IIB increases forward pulling tension in the mdx mouse. Muscle Nerve 2011, 43:694-699.

54. Wu J, Ruas JL, Estall JL, Rasbach KA, Choi JH, Ye L, Bostrom P, Tyra HM, Crawford RW, Campbell KP, Rutkowski DT, Kaufman RJ, Spiegelman BM: The unfolded protein response mediates adaptation to exercise in skeletal muscle through a PGC-1alpha/ATF6alpha complex. Cell Metab 2011, 13:160-169.

55. Sandri M: Autophagy in skeletal muscle. FEBS Lett 2010, 584:1411-1416.

56. Jovaisaite $V$, Mouchiroud $L$, Auwerx J: The mitochondrial unfolded protein response, a conserved stress response pathway with implications in health and disease. J Exp Biol 2014, 217:137-143.

57. Zhao Q, Wang J, Levichkin IV, Stasinopoulos S, Ryan MT, Hoogenraad NJ: A mitochondrial specific stress response in mammalian cells. EMBO J 2002, 21:4411-4419

58. Ju JS, Varadhachary AS, Miller SE, Weihl CC: Quantitation of "autophagic flux" in mature skeletal muscle. Autophagy 2010, 6:929-935.

59. Fader CM, Colombo MI: Autophagy and multivesicular bodies: two closely related partners. Cell Death Differ 2009, 16:70-78.

60. Francis SH, Busch JL, Corbin JD, Sibley D: cGMP-dependent protein kinases and cGMP phosphodiesterases in nitric oxide and cGMP action. Pharmacol Rev 2010, 62:525-563.

61. De Palma C, Di Paola R, Perrotta C, Mazzon E, Cattaneo D, Trabucchi E, Cuzzocrea S, Clementi E: Ibuprofen-arginine generates nitric oxide and has enhanced anti-inflammatory effects. Pharmacol Res 2009, 60:221-228.

62. Cazzato D, Assi E, Moscheni C, Brunelli S, De Palma C, Cervia D, Perrotta C, Clementi E: Nitric oxide drives embryonic myogenesis in chicken through the upregulation of myogenic differentiation factors. Exp Cell Res 2014, 320:269-280.

63. Perrotta C, Bizzozero L, Falcone S, Rovere-Querini P, Prinetti A, Schuchman EH, Sonnino S, Manfredi AA, Clementi E: Nitric oxide boosts chemoimmunotherapy via inhibition of acid sphingomyelinase in a mouse model of melanoma. Cancer Res 2007, 67:7559-7564.

64. Paolucci C, Rovere P, De Nadai C, Manfredi AA, Clementi E: Nitric oxide inhibits the tumor necrosis factor alpha -regulated endocytosis of human dendritic cells in a cyclic GMP-dependent way. J Biol Chem 2000 275:19638-19644.

65. Falcone S, Perrotta C, De Palma C, Pisconti A, Sciorati C, Capobianco A, Rovere-Querini P, Manfredi AA, Clementi E: Activation of acid sphingomyelinase and its inhibition by the nitric oxide/cyclic guanosine 3',5'-monophosphate pathway: key events in Escherichia coli-elicited apoptosis of dendritic cells. J Immunol 2004, 173:4452-4463.

66. Clementi E, Sciorati C, Nistico G: Growth factor-induced Ca2+ responses are differentially modulated by nitric oxide via activation of a cyclic GMP-dependent pathway. Mol Pharmacol 1995, 48:1068-1077.

67. Mehrpour M, Esclatine A, Beau I, Codogno P: Overview of macroautophagy regulation in mammalian cells. Cell Res 2010, 20:748-762.

68. Lokireddy S, Wijesoma IW, Teng S, Bonala S, Gluckman PD, McFarlane C, Sharma M, Kambadur R: The ubiquitin ligase Mul1 induces mitophagy in skeletal muscle in response to muscle-wasting stimuli. Cell Metab 2012, 16:613-624.

69. Schiaffino S, Dyar KA, Ciciliot S, Blaauw B, Sandri M: Mechanisms regulating skeletal muscle growth and atrophy. FEBS J 2013, 280:4294-4314.

70. Sartori R, Schirwis E, Blaauw B, Bortolanza S, Zhao J, Enzo E, Stantzou A, Mouisel E, Toniolo L, Ferry A, Stricker S, Goldberg AL, Dupont S, Piccolo S, Amthor H, Sandri M: BMP signaling controls muscle mass. Nat Genet 2013, 45:1309-1318
71. Percival JM, Anderson KN, Gregorevic P, Chamberlain JS, Froehner SC: Functional deficits in nNOSmu-deficient skeletal muscle: myopathy in nNOS knockout mice. PLoS One 2008, 3:e3387.

72. Froehner SC, Reed SM, Anderson KN, Huang PL, Percival JM: Loss of nNOS inhibits compensatory muscle hypertrophy and exacerbates inflammation and eccentric contraction-induced damage in $\mathrm{mdx}$ mice. Hum Mol Genet, in press.

73. Pallafacchina $G$, Blaauw $B$, Schiaffino S: Role of satellite cells in muscle growth and maintenance of muscle mass. Nutr Metab Cardiovasc Dis 2013, 23(Suppl 1):S12-18.

74. Sandri M, Barberi L, Bijlsma AY, Blaauw B, Dyar KA, Milan G, Mammucari C, Meskers CG, Pallafacchina G, Paoli A, Pion D, Roceri M, Romanello V, Serrano AL, Toniolo L, Larsson L, Maier AB, Munoz-Canoves P, Musaro A, Pende M, Reggiani C, Rizzuto R, Schiaffino S: Signalling pathways regulating muscle mass in ageing skeletal muscle: the role of the IGF1-Akt-mTOR-FoxO pathway. Biogerontology 2013, 14:303-323.

75. Suzuki N, Motohashi N, Uezumi A, Fukada S, Yoshimura T, Itoyama $Y$, Aoki M, Miyagoe-Suzuki Y, Takeda S: NO production results in suspensioninduced muscle atrophy through dislocation of neuronal NOS. J Clin Invest 2007, 117:2468-2476.

76. Clementi E, Brown GC, Foxwell N, Moncada S: On the mechanism by which vascular endothelial cells regulate their oxygen consumption. Proc Natl Acad Sci U S A 1999, 96:1559-1562.

77. Nisoli E, Clementi E, Paolucci C, Cozzi V, Tonello C, Sciorati C, Bracale R, Valerio A, Francolini M, Moncada S, Carruba MO: Mitochondrial biogenesis in mammals: the role of endogenous nitric oxide. Science 2003, 299:896-899.

78. Gross SS, Wolin MS: Nitric oxide: pathophysiological mechanisms. Annu Rev Physiol 1995, 57:737-769.

79. Pellegrino MW, Nargund AM, Haynes CM: Signaling the mitochondrial unfolded protein response. Biochim Biophys Acta 1833, 2013:410-416.

80. Church JE, Gehrig SM, Chee A, Naim T, Trieu J, McConell GK, Lynch GS: Early functional muscle regeneration after myotoxic injury in mice is unaffected by nNOS absence. Am J Physiol Regul Integr Comp Physiol 2011, 301:R1358-1366.

81. Samengo G, Avik A, Fedor B, Whittaker D, Myung KH, Wehling-Henricks M, Tidball JG: Age-related loss of nitric oxide synthase in skeletal muscle causes reductions in calpain S-nitrosylation that increase myofibril degradation and sarcopenia. Aging Cell 2012, 11:1036-1045.

82. Ito N, Ruegg UT, Kudo A, Miyagoe-Suzuki Y, Takeda S: Activation of calcium signaling through Trpv1 by nNOS and peroxynitrite as a key trigger of skeletal muscle hypertrophy. Nat Med 2013, 19:101-106.

83. Li D, Yue Y, Lai Y, Hakim CH, Duan D: Nitrosative stress elicited by nNOSmicro delocalization inhibits muscle force in dystrophin-null mice. J Pathol 2011, 223:88-98

84. Nguyen HX, Tidball JG: Expression of a muscle-specific, nitric oxide synthase transgene prevents muscle membrane injury and reduces muscle inflammation during modified muscle use in mice. J Physiol 2003, 550:347-356.

85. Anderson JE: A role for nitric oxide in muscle repair: nitric oxidemediated activation of muscle satellite cells. Mol Biol Cell 2000, 11:1859-1874

86. Baum O, Vieregge M, Koch P, Gul S, Hahn S, Huber-Abel FA, Pries AR, Hoppeler H: Phenotype of capillaries in skeletal muscle of nNOS-knockout mice. Am J Physiol Regul Integr Comp Physiol 2013, 304:R1175-1182

87. Thomas GD: Functional muscle ischemia in Duchenne and Becker muscular dystrophy. Front Physio/ 2013, 4:381.

88. Bredt DS: NO skeletal muscle derived relaxing factor in Duchenne muscular dystrophy. Proc Natl Acad Sci U S A 1998, 95:14592-14593.

89. Thomas GD, Sander M, Lau KS, Huang PL, Stull JT, Victor RG: Impaired metabolic modulation of alpha-adrenergic vasoconstriction in dystrophin-deficient skeletal muscle. Proc Natl Acad Sci U S A 1998 95:15090-15095.

90. Shibata K, Shimokawa H, Yanagihara N, Otsuji Y, Tsutsui M: Nitric oxide synthases and heart failure - lessons from genetically manipulated mice. J UOEH 2013, 35:147-158.

91. Lu XM, Zhang GX, Yu YQ, Kimura S, Nishiyama A, Matsuyoshi H, Shimizu J, Takaki M: The opposite roles of nNOS in cardiac ischemia-reperfusioninduced injury and in ischemia preconditioning-induced cardioprotection in mice. J Physiol Sci 2009, 59:253-262. 
92. Chao DS, Silvagno F, Bredt DS: Muscular dystrophy in mdx mice despite lack of neuronal nitric oxide synthase. J Neurochem 1998 71:784-789.

93. Crosbie RH, Straub V, Yun HY, Lee JC, Rafael JA, Chamberlain JS, Dawson VL, Dawson TM: Campbell KP: mdx muscle pathology is independent of nNOS perturbation. Hum Mol Genet 1998, 7:823-829.

doi:10.1186/s13395-014-0022-6

Cite this article as: De Palma et al:: Deficient nitric oxide signalling impairs skeletal muscle growth and performance: involvement of mitochondrial dysregulation. Skeletal Muscle 2014 4:22.

\section{Submit your next manuscript to BioMed Central and take full advantage of:}

- Convenient online submission

- Thorough peer review

- No space constraints or color figure charges

- Immediate publication on acceptance

- Inclusion in PubMed, CAS, Scopus and Google Scholar

- Research which is freely available for redistribution 